MATHEMATICS OF COMPUTATION

Volume 76, Number 258, April 2007, Pages 669-698

S 0025-5718(07)01953-9

Article electronically published on January 8, 2007

\title{
A PRIORI ESTIMATES OF SMOOTHNESS OF SOLUTIONS TO DIFFERENCE BELLMAN EQUATIONS WITH LINEAR AND QUASI-LINEAR OPERATORS
}

\author{
N. V. KRYLOV
}

\begin{abstract}
A priori estimates for finite-difference approximations for the first and second-order derivatives are obtained for solutions of parabolic equations described in the title.
\end{abstract}

\section{INTRODUCTION}

The goal of this article is to prove a priori estimates for solutions of finitedifference approximations of parabolic Bellman equations with linear and quasilinear operators. In the latter case the nonlinear operator defining the equation is still supposed to be convex with respect to the second-order derivatives of the unknown function. We present estimates for the finite-difference approximations of the first and second-order spatial derivatives. In particular, our results cover finitedifference approximations for degenerate quasi-linear parabolic equations. As far as we are aware these are the first results for such equations. The main parts of the linear and quasi-linear operators entering Bellman equations are assumed to be linear $a_{k} \Delta_{k}$ operators, that is, written as a linear combination of pure second-order derivatives in certain directions that are common to all operators. This assumption is always satisfied if the equation is uniformly nondegenerate and is generally necessary if we want to restrict ourselves to monotone difference approximations and meshes that are obtained from a fixed one by scaling (see more about it in Remark 2.4 below). Our results are valid for the usual Bellman equations and also for the optimal stopping and impulse control problems associated with them.

The motivation to obtain a priori estimates is the following. There is an approach suggested in [10], [11, and [12] to establishing the rate of convergence of $u_{h}$ to $u$ as $h \downarrow 0$, where $u$ is the true solution, $u_{h}$ the solution of finite-difference approximation of the same equation, and $h$ typically is the mesh size. Two main ideas of this approach are that the original equation and its finite-difference approximation should play symmetric roles and that one can "shake the coefficients" of the equation in order to be able to mollify under the sign of the nonlinear operator.

For elliptic Bellman equations with constant coefficients and Lipschitz free terms the first idea led to the rate of convergence of order $h^{1 / 3}$, for generic finite-difference

Received by the editor November 13, 2005 and, in revised form, May 14, 2006.

2000 Mathematics Subject Classification. Primary 65M15, 35J60, 93E20.

Key words and phrases. Finite-difference approximations, Bellman equations, fully nonlinear equations.

The work was partially supported by NSF Grant DMS-0140405. 
approximations and $h^{1 / 2}$ in the case of $a_{k} \Delta_{k}$ operators (see Remark 1.4 and Theorem 5.1 in [10], also see [2]). In contrast with the popular belief that assuming more smoothness of the data does not lead to better rates of convergence, it is proved in 5 that if the free terms are in $C^{1,1}$, then the rate is at least $h$ for the constant coefficient $a_{k} \Delta_{k}$ case and $h^{2}$ for equations with better structure.

The second idea was introduced to treat equations with variable coefficients and led to quite satisfactory error bounds for $u-u_{h}$ from an "easy" side (depending on how the equation is written this can be either the upper or lower estimate of $\left.u-u_{h}\right)$. To get an estimate from the other side on the basis of the idea of symmetry between the approximating and the original equations one needed to solve the following problem:

(P) In the case of variable coefficients estimate how much the solution of the finite-difference equation loses in the process of shaking the equation.

In the absence of a solution of the problem $(\mathrm{P})$ the idea of symmetry was still useful but only in obtaining some intermediate estimates (see, for instance, 2] and [11) and various approaches to getting the error bounds from the "hard" side were developed. In addition to the above-cited papers the interested reader should consult [3, 4, and the references therein. Note that for generic finite-difference approximations, under the assumptions of Theorem 5.3 of [1] the result of 4 is the same $h^{1 / 3}$, but the result of Theorem 5.4 of 11 is improved from $h^{1 / 21}$ to $h^{1 / 7}$. The issue of solving the problem $(\mathrm{P})$ for generic finite-difference approximations remains unsettled, and it is not clear how far off $h^{1 / 7}$ is from the true rate.

The problem $(\mathrm{P})$ was recently reduced to the problem of estimating the modulus of continuity of approximate solutions and was solved in [13 for the $a_{k} \Delta_{k}$ case in which a sharp error bound of order $h^{1 / 2}$ was obtained. The idea of symmetry worked again as in the constant coefficients case. This activity was continued in [7, where for the first time equations in domains were treated, and in [6], where under various smoothness assumptions the rates $h^{1 / 2}, h$, and $h^{2}$ were obtained for linear degenerate equations of $a_{k} \Delta_{k}$ form. For the linear case the rate $h^{1 / 2}$ was earlier obtained in [8] by a method close to a method from [12] (Lemma 5.1 of [8] is a version of Theorem 2.1 of [12]). However, this method does not allow one to get rates $h$ and $h^{2}$.

The main technical result of 13 is the a priori estimate of the derivative of $u_{h}$ with respect to $x$ stated as Theorem 5.2 and proved by quite subtle estimates. It turns out that there is a much easier method to prove Theorem 5.2 of [13] which in addition carries over to much more general equations with quasi-linear operators and to obtaining estimates for the second-order finite differences of $u_{h}$. The method is almost as simple as the one used in [6] for linear equations.

We present this new method here and concentrate only on a priori estimates to keep the article within reasonable limits. Once the a priori estimates are obtained, one can follow familiar patterns to get error bounds in the various cases of linear or quasi-linear operators, degenerate or weakly nondegenerate or else uniformly nondegenerate, with $C^{1}$ or $C^{1,1}$ coefficients. In particular, we hope to obtain first estimates on the rate of convergence in the case of degenerate quasi-linear operators with Lipschitz coefficients. Our preliminary computations also show that under the assumptions of Theorem 2.12 the estimate $\left|u-u_{h}\right| \leq N h^{2 / 3}$ holds in the elliptic case. These and some other possible applications indicated below of our results, we intend to develop in the future. 
Hongjie Dong and the referees of the paper made valuable comments on the first version of it for which the author is sincerely grateful.

\section{Setting And main Results}

Our first few results concern equations of the type

$$
F\left(\delta_{\tau}^{T} u, \Delta_{h, \ell_{k}} u, \delta_{h, \ell_{k}} u, u\right)=0,
$$

where

$$
\begin{aligned}
& F\left(\phi, q_{k}, p_{k}, \psi\right)=F(\phi, q, p, \psi, t, x) \\
& =\sup _{\alpha \in A}\left[r^{\alpha}(t) \phi+\sum_{|k|=1}^{d_{1}}\left(a_{k}^{\alpha}(t, x) q_{k}+b_{k}^{\alpha}(t, x) p_{k}\right)-c^{\alpha}(t, x) \psi+f^{\alpha}(p, \psi, t, x)\right],
\end{aligned}
$$

$\delta_{\tau}^{T} u, \Delta_{h, \ell_{k}} u, \delta_{h, \ell_{k}} u$ are finite-difference approximations of the time derivative, the pure second-order derivative in the direction $\ell_{k}$, and the first-order derivative in the direction $\ell_{k}$, respectively. Detailed descriptions of the above objects now follow.

Let $A$ be a separable metric space, $d, d_{1} \geq 1$ integers, and let

$$
r^{\alpha}=r^{\alpha}(t), \quad a_{k}^{\alpha}=a_{k}^{\alpha}(t, x), \quad b_{k}^{\alpha}=b_{k}^{\alpha}(t, x), \quad c^{\alpha}=c^{\alpha}(t, x)
$$

be real-valued bounded functions of $(\alpha, t, x)$ defined on $A \times \mathbb{R} \times \mathbb{R}^{d}$ for $k= \pm 1, \ldots, \pm d_{1}$. Also let some vectors $\ell_{k} \in \mathbb{R}^{d}$ be defined for $k= \pm 1, \ldots, \pm d_{1}$ and let

$$
T, h_{0} \in(0, \infty), \quad \delta \in(0,1], \quad K_{0}, K_{1}, K_{2}, K_{3} \in[0, \infty), \quad m \in \mathbb{R}
$$

be some constants fixed throughout the article. It is worth noting that $\ell_{k}, k=$ $\pm 1, \ldots, \pm d_{1}$, are not supposed to form a basis in $\mathbb{R}^{d}$ or even generate $\mathbb{R}^{d}$. This becomes crucial when one proves the estimates of the first-order differences of solutions with respect to parameters on which the coefficients may depend. Notice also that the lengths of the $\ell_{k}$ 's can be different and some of them can be just zero (and we will use this possibility later). The constant $T$ gives us the time interval $[0, T)$ on which the equation is investigated, $h_{0}$ "calibrates" the mesh-sizes in the $x$ variable, and the constant $\delta$ will appear in various requirements of nondegeneracy. The constant $K_{0}$ is the most basic one; it is used in formulations of the very basic assumptions. The constant $K_{1}$ is used to control either the maximum magnitude of the solution or its oscillation. The constant $K_{2}$ will appear in our assumption on the growth of $f$ with respect to the "gradient" of the solution (see Assumption 2.5 (ii), which looks very much like the one commonly used in the theory of quasi-linear PDEs. By the way, the author's efforts to use Assumption 2.5(iii), stated similarly, failed.) The constant $K_{3}$ is used to control various quantities having lesser impact on our results than those controlled by $K_{0}, K_{1}, K_{2}$. Finally, the constant $m$ is used to extract various results, which one gets in the theory of parabolic PDEs after replacing $u(t, x)$ with $u(t, x) e^{m t}$.

For any vector $l \in \mathbb{R}^{d}, \eta, \tau>0$, and a function $u$, introduce

$$
\begin{gathered}
\delta_{\eta, l} u(x)=\frac{u(x+\eta l)-u(x)}{\eta}, \quad \tau_{T}(t)=\tau \wedge(T-t)^{+}, \\
\delta_{\tau}^{T} u(t, x)=\frac{u\left(t+\tau_{T}(t), x\right)-u(t, x)}{\tau}, \quad \delta_{\tau} u(t, x)=\frac{u(t+\tau, x)-u(t, x)}{\tau}, \\
\Delta_{\eta, l} u(x)=\frac{u(x+\eta l)-2 u(x)+u(x-\eta l)}{\eta^{2}},
\end{gathered}
$$


where the notation $a^{ \pm}=(1 / 2)(|a| \pm a)$ is used. Observe that with the above definition of $\delta_{\tau}^{T}$ equation (2.1) makes perfect sense for $t<T$ for functions $u(t, x)$ defined only for $t \leq T$. We do not need to extend $u$ beyond $T$ in order to compute the finite-difference approximation of its derivative in time for $t<T$.

Assumption 2.1. (i) The functions $r^{\alpha}, a_{k}^{\alpha}, b_{k}^{\alpha}$, and $c^{\alpha}$ are continuous with respect to $\alpha$;

(ii) the functions $b_{k}^{\alpha}$ satisfy the Lipschitz condition with constant $K_{0}$ with respect to $x$;

(iii) the function $c^{\alpha}$ satisfies the Lipschitz condition with constant $K_{3}$ with respect to $x$;

(iv) we have

$$
\ell_{-k}=-\ell_{k}, \quad a_{-k}^{\alpha}=a_{k}^{\alpha}, \quad\left|\ell_{k}\right| \leq K_{0}, \quad r_{k}^{\alpha} \geq 0, \quad a_{k}^{\alpha} \geq 0
$$

(for all values of the arguments and $k$ ).

An important feature of Assumption 2.1 is that no control on the sizes of $r^{\alpha}$, $a_{k}^{\alpha}, b_{k}^{\alpha}$, and $c^{\alpha}$ is imposed (however, remember that from the very beginning they are assumed to be bounded).

Assumption 2.2. For any unit $l \in \mathbb{R}^{d}$ and $\eta>0$, we have

$$
\left|\delta_{\eta, l} a_{k}^{\alpha}\right| \leq K_{0}\left(\sqrt{a_{k}^{\alpha}}+\eta\right)
$$

Remark 2.1. It is easy to see that Assumption 2.2 is satisfied (with, perhaps, different $K_{0}$ ) if and only if $\sigma_{k}^{\alpha}:=\sqrt{a_{k}^{\alpha}}$ satisfies the Lipschitz condition with constant $K_{0}$ with respect to $x$.

Indeed, the necessity follows after letting $\eta \downarrow 0$ and the sufficiency is a direct consequence of the formula

$$
\delta_{\eta, l} a_{k}^{\alpha}=2 \sigma_{k}^{\alpha} \delta_{\eta, l} \sigma_{k}^{\alpha}+\eta\left(\delta_{\eta, l} \sigma_{k}^{\alpha}\right)^{2} .
$$

Below we are also using the well-known fact that a continuous function $v(x)$ is Lipschitz continuous with constant $K$ if and only if its generalized gradient $v_{x}=$ $D_{x} v$ satisfies $\left|v_{x}\right| \leq K$ (a.e.).

Definition 2.2. Let $B$ be a finite subset of $\mathbb{R}^{d}$ and $p(x, y)$ a real-valued function on $\mathbb{R}^{d} \times \mathbb{R}^{d}$. For an $x_{0} \in \mathbb{R}^{d}$ we say that the operator

$$
S: u \rightarrow S u, \quad S u(x)=\sum_{y \in B \cup\{0\}} p(x, x+y) u(y)
$$

respects the maximum principle at $x_{0}$ relative to $B$ if, for any function $\phi(x)$ such that $\phi\left(x_{0}+y\right) \geq \phi\left(x_{0}\right)$ for all $y \in B$, we have $S u\left(x_{0}\right) \geq 0$.

Obviously, the operators $\delta_{\eta, l}$ and $\Delta_{\eta, l}$ respect the maximum principle at any point relative to appropriate sets.

For $h>0$ set

$$
L_{h}^{\alpha} u=a_{k}^{\alpha} \Delta_{h, \ell_{k}} u+b_{k}^{\alpha} \delta_{h, \ell_{k}} u-c^{\alpha} u,
$$

where and throughout the paper the summation convention is enforced. For each $t$ the operator $L_{h}^{\alpha}=L_{h}^{\alpha}(t, x)$ can be considered as an operator on functions defined on $\mathbb{R}^{d}$.

Assumption 2.3. We have $a_{k}^{\alpha} \geq h_{0}\left(b_{k}^{\alpha}\right)^{-}($recall (2.3) $)$. 
Remark 2.3. It is easy to see that Assumption 2.3 implies that for $h \in\left(0, h_{0}\right], t \in \mathbb{R}$, and $\alpha \in A$ the operator $L_{h}^{\alpha}(t, x)+c^{\alpha}(t, x)$ respects the maximum principle at any point $x_{0}$ relative to $\Lambda_{0}$, where

$$
\Lambda_{0}:=\left\{h \ell_{k}: k= \pm 1, \ldots, \pm d_{1}\right\} .
$$

In turn, provided that all $\ell_{k}$ are different, the said property of $L_{h}^{\alpha}(t, x)+c^{\alpha}(t, x)$ implies what is required in Assumption 2.3 .

To satisfy Assumption 2.3 it is sufficient to require that $b_{k}^{\alpha} \geq 0$, in which case what we use is just an upwind discretization of the "transportation" term.

Remark 2.4. The operators $L_{h}^{\alpha}$ are natural approximations of the operator

$$
L^{\alpha} u=a_{k}^{\alpha} \ell_{k}^{i} \ell_{k}^{j} u_{x^{i} x^{j}}+b_{k}^{\alpha} \ell_{k}^{i} u_{x^{i}}-c u
$$

in the sense that $L_{h}^{\alpha} u \rightarrow L^{\alpha} u$ as $h \downarrow 0$ for all smooth $u$.

One may wonder how wide is the class of operators given in the usual form

$$
L u=a^{i j} u_{x^{i} x^{j}}+b^{i} u_{x^{i}}
$$

which admit such a special approximation. We discuss this issue in Section 9 ,

Next, we describe the free term in the equation, which is given by a real-valued function

$$
f^{\alpha}=f^{\alpha}(p, \psi, t, x)
$$

defined on $A \times \mathbb{R}^{2 d_{1}} \times \mathbb{R} \times \mathbb{R} \times \mathbb{R}^{d}$.

Assumption 2.4. The function $f^{\alpha}$ is bounded, $f^{\alpha}$ is continuous in $\alpha$, continuous in $(p, \psi, x)$ and, for any $\alpha$ and $t$, its generalized gradients $D_{p} f^{\alpha}, D_{\psi} f^{\alpha}$, and $D_{x} f^{\alpha}$ in $p, \psi$, and $x$, respectively, satisfy

$$
\left|D_{p^{k}} f^{\alpha}\right| \leq K_{0} \sqrt{a_{k}^{\alpha}}, \quad k= \pm 1, \ldots, \pm d_{1}, \quad\left|D_{\psi} f^{\alpha}\right| \leq K_{0}, \quad\left|D_{x} f^{\alpha}\right| \leq K_{3}
$$

for almost all $(p, \psi, x) \in \mathbb{R}^{2 d_{1}} \times \mathbb{R} \times \mathbb{R}^{d}$.

For fixed $h, \tau>0$ we consider the equation

$$
\sup _{\alpha \in A}\left[r^{\alpha} \delta_{\tau}^{T} u+L_{h}^{\alpha} u+g^{\alpha}\right]=0
$$

where

$$
g^{\alpha}=g^{\alpha}(t, x)=f^{\alpha}\left(\delta_{h, \ell_{k}} u(t, x), u(t, x), t, x\right) .
$$

Observe that equation (2.9) takes the form (2.1). The presence of $r^{\alpha}$ in these equations allows us to treat the normalized Bellman equations (see [9]), which arise, for instance, in optimal stopping problems or problems with singular control.

Fix a vector $l \in \mathbb{R}^{d}$ with $|l| \leq K_{0}$ and a number $\eta \in(0, h]$. Set

$$
h_{i}=h \quad \text { for } \quad|i|=1, \ldots, d_{1}, \quad h_{d+1}=h_{-(d+1)}=\eta, \quad \ell_{d+1}=-\ell_{-(d+1)}=l,
$$

$$
\Lambda=\left\{h_{1} \ell_{ \pm 1}, \ldots, h_{d+1} \ell_{ \pm\left(d_{1}+1\right)}\right\} .
$$

We treat $\Lambda_{0}$ as a list rather than a set with specified elements, even if $\ell_{1}=\ell_{2}$ we include in the list this vector twice. 
Observe that in (2.5) only $\ell_{k} \in \Lambda_{0}$ are involved. However, the method of "shaking" the coefficients requires estimates of difference derivatives in all directions and not only along the mesh. This is the reason why we introduce $\Lambda$. Set

$$
\begin{aligned}
& \Lambda_{n}=\sum_{1}^{n} \Lambda=\left\{x: x=l_{1}+\ldots+l_{n}, l_{1}, \ldots, l_{n} \in \Lambda\right\}, \quad \Lambda_{\infty}=\bigcup_{n} \Lambda_{n}, \\
& \overline{\mathcal{M}}_{T}=\{(n \tau) \wedge T: n=0,1, \ldots\} \times \Lambda_{\infty}, \quad \mathcal{M}_{T}=\overline{\mathcal{M}}_{T} \cap\left([0, T) \times \mathbb{R}^{d}\right) .
\end{aligned}
$$

Fix a finite set $Q \subset \mathcal{M}_{T}$, assume that

$$
\left.Q\right|_{0}:=Q \cap\left(\{0\} \times \mathbb{R}^{d}\right) \neq \emptyset
$$

and define

$$
\begin{gathered}
\bar{Q}=Q \cup\left\{\left(t+\tau_{T}(t), x\right):(t, x) \in Q, t+\tau_{T}(t)=T\right\}, \\
Q_{1}^{o}=\left\{(t, x) \in Q: t<T,\left(t+\tau_{T}(t), x\right) \in \bar{Q},(t, x+\Lambda) \subset Q\right\}, \\
\partial_{1} Q=\bar{Q} \backslash Q_{1}^{o} .
\end{gathered}
$$

Obviously, it may happen that $\bar{Q}=Q$. The subscript 1 is used above because later on we will need a "fatter" boundary $\partial_{2} Q$.

Finally, define $T^{\prime}$ as the least $n \tau, n=1,2, \ldots$, such that $n \tau \geq T$, recall that $m \in \mathbb{R}($ see (2.3) $)$ is a given fixed constant and introduce

$$
\begin{gathered}
\xi(t)=e^{m t}, \quad t<T, \quad \xi(T)=e^{m T^{\prime}}, \quad \xi_{(+)}=\xi \vee 1, \quad \xi_{(-)}=\xi \wedge 1, \\
c_{m}=\frac{1-e^{-m \tau}}{\tau}, \quad \lambda=\inf _{\alpha, t, x}\left[c^{\alpha}(t, x)+r^{\alpha}(t) c_{m}\right] .
\end{gathered}
$$

Introducing a discontinuous function $\xi(t)$ may look unnatural. However, what is important for us is that

$$
\xi \delta_{\tau}^{T} u=e^{-m \tau} \delta_{\tau}^{T}(\xi u)-c_{m}(\xi u)
$$

on $\mathcal{M}_{T}$ for any $u=u(t, x)$.

Everywhere below in this section $u$ is a given function on $\overline{\mathcal{M}}_{T}$ satisfying (2.9) in $Q$. In our first result no control on the sizes of $r^{\alpha}, a_{k}^{\alpha}, b_{k}^{\alpha}$, and $c^{\alpha}$ is imposed.

Theorem 2.5. Let $h \in\left(0, h_{0}\right]$. Then, under Assumptions 2.1 through 2.4 there are constants $N=N\left(d_{1}, K_{0}\right), N^{*}=N^{*}\left(d_{1}, K_{0}, K_{3}\right)$ such that if $\lambda \geq N$, then on $\left.Q\right|_{0}$,

$$
\left|\delta_{\eta, l} u\right| \leq N^{*} e^{m^{+}(T+\tau)}\left[1+\max _{\bar{Q}}\left|\xi_{(-)} u\right|+\max _{k, \partial_{1} Q}\left(\left|\xi_{(-)} \delta_{h, \ell_{k}} u\right|+\left|\xi_{(-)} \delta_{\eta, l} u\right|\right)\right] .
$$

We prove this theorem in Section 4

Remark 2.6. This theorem is similar to Theorem 5.2 of [13] and entails all the consequences derived from the latter in [13] and [7. In particular, by using Theorem 5.6 of [13] and comparing the equations for $u$ and $u(\tau+\cdot, \cdot)$ an estimate of $\delta_{\tau} u$ can be obtained if we require the data to have bounded derivatives in $t$.

Remark 2.7. Theorem 2.5 has an immediate application to elliptic equations. In that case $u$ is independent of $t$, one can take $r^{\alpha} \equiv 0$, and use as large a negative $m$ as one wishes without affecting $\lambda$. Then it is seen that in (2.16) the maximums over $\bar{Q}$ and $\partial_{1} Q$ reduce to the maximums over $\left.Q\right|_{0}$ and $\left.Q\right|_{0} \cap \partial_{1} Q$, respectively. 
Our second result concerns Bellman equations with more general quasi-linear operators. This time (2.9) is assumed to be uniformly nondegenerate in the space generated by the $\ell_{k}$ 's. We will allow $f^{\alpha}(p, \psi, t, x)$ to grow quadratically with respect to $p$, and therefore no $b_{k}^{\alpha}$ are needed. The term $c^{\alpha} u$ also could be absorbed in $f^{\alpha}$. However, we keep it, in order to state Theorem 2.11 in a simpler way.

Assumption 2.5. (i) The functions $a_{k}^{\alpha}$ also depend on $\psi$ :

$$
a_{k}^{\alpha}=a_{k}^{\alpha}(\psi, t, x)
$$

and equation (2.1) holds in $Q$, where $F$ is defined by (2.2) with $a_{k}^{\alpha}(\psi, t, x)$ in place of $a_{k}^{\alpha}(t, x)$. The functions $a_{k}^{\alpha}(\psi, t, x)$ are Lipschitz continuous in $x$ with constant $K_{3}$, Lipschitz continuous in $\psi$ with a constant $\omega \in(0, \infty)$,

$$
a_{k}^{\alpha} \geq \delta, \quad|k| \leq d_{1}, \quad c^{\alpha} \geq-K_{3} .
$$

(ii) The function $f^{\alpha}$ is continuous in $\alpha$, continuous in $(p, \psi, x)$, and for all values of the arguments, satisfying $|\psi| \leq K_{1}$ and $|p| \geq K_{2}$, it holds that

$$
\left|f^{\alpha}\right| \leq \omega|p|^{2}+K_{3} \text {. }
$$

(iii) For each $\alpha$ and $t$ the generalized gradients $D_{p} f^{\alpha}, D_{\psi} f^{\alpha}$, and $D_{x} f^{\alpha}$ of $f^{\alpha}$ with respect to $p, \psi$, and $x$, respectively, satisfy

$$
\left|D_{p} f^{\alpha}\right| \leq \omega|p|+K_{3}, \quad\left|D_{\psi} f^{\alpha}\right| \leq \omega|p|^{2}+K_{3},
$$

$$
\left|D_{x} f^{\alpha}\right| \leq \omega|p|^{3}+K_{3}
$$

(a.e.) on the set $\left\{|\psi| \leq K_{1}\right\}$.

Remark 2.8. Clearly, Assumption 2.5 (ii) is satisfied with any $\omega>0$ and appropriate $K_{3}(\omega)$ if

$$
\sup _{\alpha, t, x, \psi}\left|f^{\alpha}(p, \psi, t, x)\right|=o\left(|p|^{2}\right)
$$

as $|p| \rightarrow \infty$. This includes all functions affine in $p$ provided that the coefficients are bounded. A similar situation occurs with Assumption 2.5 (iii).

Assumption 2.6. For a constant $C \geq 4$ depending only on $d_{1}$, the exact value of which can be determined by examining the proof of Theorem 2.9, we have

$$
C K_{1}\left(1+K_{1}\right) \omega \leq \delta .
$$

Theorem 2.9. Let $b_{k}^{\alpha} \equiv 0$ and let Assumptions 2.1, 2.5, and 2.6 be satisfied. Assume that $|u| \leq K_{1}$ in $\bar{Q}$ and $\left|\delta_{h, \ell_{k}} u\right| \leq K_{3}$ on $\partial_{1} Q$ if $|k| \leq d_{1}$. Then in $\bar{Q}$,

$$
\left|\delta_{h, \ell_{k}} u\right| \leq N=N\left(d_{1}, \delta, K_{1}, K_{2}, K_{3}\right), \quad|k| \leq d_{1}
$$

In particular, $N$ is independent of $T$.

The proof of this theorem is given in Section 5 .

Remark 2.10. If $\omega$ is large, we need $K_{1}$ to be small in order to satisfy (2.18), that is, we need $u$ to be small. By replacing $u$ with $u-\gamma$, where $\gamma$ is any constant, we see that, actually, we need the oscillation of $u$ rather than $u$ itself to be small if $\omega$ is not. This restriction could be completely avoided if we proved an interior version of Theorem 2.9 and a priori Hölder continuity of $u$. It seems to the author that this is possible, but requires much more work. 
Theorem 2.11. Under the assumptions of Theorem 2.9 suppose that $\left|\delta_{\eta, l} u\right| \leq K_{3}$ on $\partial_{1} Q$ and $a_{k}^{\alpha}$ are independent of $\psi$. Then there is a constant $N=N\left(d_{1}, \delta, K_{1}, K_{2}\right.$, $\left.K_{3}\right)$, such that if $\lambda \geq N$, then on $\left.Q\right|_{0}$,

$$
\left|\delta_{\eta, l} u\right| \leq N e^{m^{+}(T+\tau)} .
$$

This is a simple corollary of Theorems 2.9 and 2.5 with $h_{0}=h$ in the latter. Indeed, once we know that the values of $\left|\delta_{h, \ell_{k}} u\right|$ and $|u|$ are dominated by a constant, the behavior of $f^{\alpha}(p, \psi, t, x)$ for large $|p|$ becomes irrelevant and we can even multiply it by an appropriate cut-off function in such a way that the new $f^{\alpha}$ would satisfy Assumption 2.4 and $u$ would still satisfy the new equation.

Our next result is about second-difference estimates.

Assumption 2.7. (i) The function $f^{\alpha}$ is independent of $p$ and $\psi$.

(ii) For any $i, j= \pm 1, \ldots, \pm\left(d_{1}+1\right)$ and $\psi$ standing for any of the functions $b_{k}^{\alpha}$, $c^{\alpha}$, and $f^{\alpha}$ we have

$$
\left|\delta_{h_{j}, \ell_{j}} \delta_{h_{i}, \ell_{i}} \psi\right| \leq K_{3}, \quad\left|\delta_{h_{i}, \ell_{i}} f^{\alpha}\right| \leq K_{3}, \quad\left|\delta_{h_{j}, \ell_{j}} \delta_{h_{i}, \ell_{i}} a_{k}^{\alpha}\right| \leq K_{0}+K_{3} \sqrt{a_{k}^{\alpha}} .
$$

A typical case when the third inequality in (2.19) is satisfied occurs if $a_{k}^{\alpha}=\left(\sigma_{k}^{\alpha}\right)^{2}$, where $\sigma_{k}^{\alpha}$ is bounded and twice continuously differentiable.

In contrast with the above results in which no control on the magnitudes of $r^{\alpha}$, $a_{k}^{\alpha}, b_{k}^{\alpha}$, and $c^{\alpha}$ is required, this time we need the following.

Assumption 2.8. We have

$$
\delta \leq \sup _{\alpha \in A} a_{k}^{\alpha} \leq K_{0}, \quad r^{\alpha},\left|b_{k}^{\alpha}\right|,\left|c^{\alpha}\right|,\left|f^{\alpha}\right| \leq K_{3} .
$$

The following assumption is about a special structure of the set of our basic vectors $\ell_{k}, k= \pm 1, \ldots, \pm d_{1}$. For $d_{1}=2$ and the standard grid (generated by $\pm e_{1}, \pm e_{2}$ ) it means that this set contains all eight neighboring points of the origin on the grid.

Assumption 2.9. There exists an integer $1 \leq d_{0}<d_{1}$ such that for the list

$$
\mathcal{L}:=\left\{h \ell_{ \pm 1}, \ldots, h \ell_{ \pm d_{0}}\right\}
$$

and any $\ell_{k}$ with $d_{1}<|k| \leq d_{1}$ there exist $l_{1}, l_{2} \in \mathcal{L}$ such that

$$
l_{1} \neq l_{2}, \quad l_{1} \neq-l_{2}, \quad \ell_{k}=l_{1}+l_{2} .
$$

One may think that Assumption 2.9 excludes the equations with only one spatial variable, where it is natural to take $d_{1}=1$ and $\Lambda_{0}=\left\{\ell_{1},-\ell_{1}\right\}$. However, we do not require $\ell_{k}$ to be nonzero, and one can take $\Lambda_{0}$ to be $\left\{\ell_{1},-\ell_{1}, \ell_{2},-\ell_{2}, \ell_{3},-\ell_{3}\right\}$ with $\ell_{2}=0$ and $\ell_{3}=\ell_{1}$. In that case Assumption 2.9 is satisfied with $\mathcal{L}=$ $\left\{\ell_{1},-\ell_{1}, \ell_{2},-\ell_{2}\right\}$. By the way the fact that now the origin is one of the $\ell_{k}$ in no way contradicts Assumption 2.8, because in that case $\delta_{h, \ell_{k}} \phi=0$ and one can assign any value to $a_{k}^{\alpha}$ without changing the equation.

Define

$$
\begin{gathered}
Q_{2}^{o}=\left\{(t, x) \in Q: t<T,\left(t+\tau_{T}(t), x\right) \in \bar{Q},\left(t, x+\Lambda_{0}+\Lambda_{0}\right) \subset Q\right\}, \\
\partial_{2} Q=Q \backslash Q_{2}^{o} .
\end{gathered}
$$

Here, naturally, $x+\Lambda_{0}+\Lambda_{0}=\left\{x+y+z: y, z \in \Lambda_{0}\right\}$. 
Theorem 2.12. Suppose that Assumptions 2.1, 2.2, 2.3, 2.7,2.9 are satisfied. Then there exists a constant $N=N\left(\delta, d_{1}, K_{0}\right)$ such that if $\lambda \geq N$, then in $\left.Q\right|_{0}$ for $i, j= \pm 1, \ldots, \pm d_{1}$ we have

$$
\left|\delta_{h, \ell_{j}} \delta_{h, \ell_{i}} u\right| \leq N^{*} e^{m^{+}(T+\tau)} R,
$$

where $N^{*}=N^{*}\left(h_{0}, \delta, d_{1}, K_{0}, K_{3}\right)$,

$$
\begin{gathered}
R=1+\max _{\bar{Q}}\left|\xi_{(-)} u\right|+\max _{Q}\left(\xi_{(-)} \delta_{\tau}^{T} u\right)^{-} \\
+\max _{|i|,|j| \leq d_{1}} \max _{\partial_{2} Q}\left|\xi_{(-)} \delta_{h, \ell_{i}} \delta_{h, \ell_{j}} u\right|+\max _{|i| \leq d_{1}} \max _{\bar{Q}}\left|\xi_{(-)} \delta_{h, \ell_{i}} u\right| .
\end{gathered}
$$

This theorem is proved in Section 7 following a quite long Section 6 that contains the proof of Theorem 2.12 under additional assumptions.

Remark 2.13. To get "closed" estimates of $\delta_{h, \ell_{j}} \delta_{h, \ell_{i}} u$ we need to exclude $\delta_{h, \ell_{i}} u$ and $\delta_{\tau}^{T} u$ from $R$. This can be done by using Theorem 2.5 and the idea from Remark 2.6. Another situation when $\delta_{\tau}^{T} u$ drops out presents when $u$ is independent of $t$, so that, actually, we are dealing with elliptic equations. We say more about this in the comments after Theorem 2.14

In the case of $a_{k}^{\alpha}$ independent of $x$, Assumption 2.9 is not needed.

Theorem 2.14. Suppose that Assumptions 2.1, 2.2, 2.3, 2.7, and 2.8 are satisfied. Also assume that the $a_{k}^{\alpha}$ are independent of $x,\left|\delta_{h_{i}, \ell_{i}} b_{k}^{\alpha}\right| \leq K_{3} \sqrt{a_{k}^{\alpha}},|i| \leq d_{1}+1$, $|k| \leq d_{1}$, and $\lambda>0$. Then in $\left.Q\right|_{0}$ for $k= \pm 1, \ldots, \pm d_{1}$ we have

$$
\left|\Delta_{h, \ell_{k}} u\right| \leq N^{*} e^{m^{+}(T+\tau)} R_{0}, \quad\left(\Delta_{\eta, l} u\right)^{-} \leq N^{*} e^{m^{+}(T+\tau)} R,
$$

where $N^{*}=N^{*}\left(\lambda, h_{0}, \delta, d_{1}, K_{3}\right)$,

$$
\begin{gathered}
R_{0}=1+\max _{\bar{Q}}\left|\xi_{(-)} u\right|+\max _{Q}\left(\xi_{(-)} \delta_{\tau}^{T} u\right)^{-} \\
+\max _{|i| \leq d_{1}} \max _{\partial_{1} Q}\left|\xi_{(-)} \Delta_{h_{i}, \ell_{i}} u\right|+\max _{|i| \leq d_{1}} \max _{\bar{Q}}\left|\xi_{(-)} \delta_{h_{i}, \ell_{i}} u\right|,
\end{gathered}
$$

and $R$ is obtained from $R_{0}$ by taking $d_{1}+1$ in place of $d_{1}$.

This theorem, proved in Section 8 , is a direct generalization of the corresponding result from [5]: lower-order coefficients are allowed to depend on $(t, x)$ and we consider parabolic equations. In connection with the latter observe that if $r^{\alpha} \equiv 0$ (elliptic case), then one can let $m \rightarrow-\infty$ and see that in the definitions of $R_{0}$ and $R$ one can replace $\partial_{1} Q$ with $\left.Q\right|_{0} \cap \partial_{1} Q$.

\section{Some technical TOOLS}

For any $\eta \in \mathbb{R}^{d}, \nu \geq 0$ set

$$
T_{\nu, \eta} \psi(x):=\psi(x+\nu \eta) .
$$

Lemma 3.1. For any $\nu>0, l_{1}, l_{2} \in \mathbb{R}^{d}$, and functions $a(x), \psi(x)$,

$$
\begin{aligned}
\delta_{\nu, l_{1}}(a \psi)= & \left(\delta_{\nu, l_{1}} a\right) \psi+\left(T_{\nu, l_{1}} a\right) \delta_{\nu, l_{1}} \psi=a \delta_{\nu, l_{1}} \psi+\psi \delta_{\nu, l_{1}} a+\nu\left(\delta_{\nu, l_{1}} a\right) \delta_{\nu, l_{1}} \psi \\
\delta_{\nu, l_{2}} \delta_{\nu, l_{1}}(a \psi)= & a \delta_{\nu, l_{2}} \delta_{\nu, l_{1}} \psi+\left(\delta_{\nu, l_{2}} a\right) \delta_{\nu, l_{1}} \psi+\left(\delta_{\nu, l_{1}} a\right) \delta_{\nu, l_{2}} \psi \\
& +h\left[\delta_{\nu, l_{1}} a+\delta_{\nu, l_{2}} a\right] \delta_{\nu, l_{2}} \delta_{\nu, l_{1}} \psi+\left(\delta_{\nu, l_{2}} \delta_{\nu, l_{1}} a\right) T_{h, l_{1}+l_{2}} \psi \\
\Delta_{\nu, l_{1}}(a \psi)= & a \Delta_{\nu, l_{1}} \psi+\psi \Delta_{\nu, l_{1}} a+\left(\delta_{\nu, l_{1}} a\right) \delta_{\nu, l_{1}} \psi+\left(\delta_{\nu,-l_{1}} a\right) \delta_{\nu,-l_{1}} \psi
\end{aligned}
$$


In particular,

$$
\Delta_{\nu, l_{1}}\left(\psi^{2}\right)=2 \psi \Delta_{\nu, l_{1}} \psi+\left(\delta_{\nu, l_{1}} \psi\right)^{2}+\left(\delta_{\nu,-l_{1}} \psi\right)^{2} .
$$

This lemma is proved by straightforward computations (cf. [13]). In the following lemma we use Definition 2.2 .

Lemma 3.2. If an operator

$$
S \psi(x)=\sum_{y \in \Lambda \cup\{0\}} p(x, x+y) \psi(x+y)
$$

respects the maximum principle at a point $x_{0} \in \mathbb{R}^{d}$ relative to $\Lambda$ and $\psi$ is a function such that $\psi\left(x_{0}\right) \leq 0$, then $-S \psi \leq S\left(\psi^{-}\right)$at $x_{0}$. In particular, $\phi^{-} S \phi^{-} \geq-\phi^{-} S \phi$ at $x_{0}$ for any function $\phi$.

This follows from the definition and the fact that $\psi+\psi^{-} \geq 0$ on $x_{0}+\Lambda$ and $\psi+\psi^{-}=0$ at $x_{0}$.

The following lemma from [13] is used in the proof of Theorem 2.12 ,

Lemma 3.3. Let $\psi$ be a function on $\mathbb{R}^{d}, \nu>0$. Then

$$
\left|\Delta_{\nu, \eta} \psi\right| \leq\left|\delta_{\nu,-\eta}\left(\left(\delta_{\nu, \eta} \psi\right)^{-}\right)\right|+\left|\delta_{\nu, \eta}\left(\left(\delta_{\nu,-\eta} \psi\right)^{-}\right)\right| .
$$

\section{Proof of Theorem 2.5}

We start with some preparations. From now on index $k$ will run through $\left\{ \pm 1, \ldots, \pm d_{1}\right\}$ and $i, j$ through $\left\{ \pm 1, \ldots, \pm\left(d_{1}+1\right)\right\}$. By $N$ and $N^{*}$ in this section we denote generic constants depending on the data as in the statement of the theorem. We use the notation (2.11) through (2.14) and introduce a few new objects. We need two constants $\varepsilon$ and $\mu$ defined by

$$
\varepsilon^{-1}-2 \varepsilon d_{1}=1, \quad 4 \mu=\left(d_{1}+1\right)^{-1} \wedge \varepsilon .
$$

Introduce

$$
\begin{aligned}
& \Gamma=\left\{\gamma=\left(\gamma_{i}: i= \pm 1, \ldots, \pm\left(d_{1}+1\right)\right): \gamma_{i} \in\left[\varepsilon, \varepsilon^{-1}\right]\right\} \\
& \delta_{i}=\delta_{h_{i}, \ell_{i}}, \quad P_{\gamma} \phi=\gamma_{i} \delta_{i} \phi, \quad v=\xi u, \quad \Delta_{k}=\Delta_{h_{k}, \ell_{k}} .
\end{aligned}
$$

By using (2.15) we see that in $Q$,

$$
F\left(e^{-m \tau} \delta_{\tau}^{T} v-c_{m} v, \xi \Delta_{k} u, \xi \delta_{k} u, \xi u\right)=0 .
$$

Also introduce

$$
\begin{gathered}
v_{\gamma}=P_{\gamma} v, \quad v_{i}=\delta_{i} v \\
P_{\gamma \mu} \phi=v_{\gamma}^{-} P_{\gamma} \phi-\mu v_{i} \delta_{i} \phi \\
W=\sum_{i} v_{i}^{2}, \quad V_{\gamma \mu}=\left[v_{\gamma}^{-}\right]^{2}+\mu W .
\end{gathered}
$$

Observe that

$$
P_{\gamma \mu} v=-V_{\gamma \mu} .
$$

Finally, let $\left(\gamma_{0}, t_{0}, x_{0}\right) \in \Gamma \times \bar{Q}$ be a point at which $V_{\gamma \mu}$ attains its maximum value over $\Gamma \times \bar{Q}$.

Theorem 4.1. The assertions of Theorem 2.5 hold true if, in addition to its assumptions, $\left(t_{0}, x_{0}\right) \in Q_{1}^{o}$ and

$$
v_{\gamma_{0}}^{-}\left(t_{0}, x_{0}\right) \geq(1 / 2) \max _{\bar{Q}, i}\left|v_{i}\right|
$$


To prove this theorem we need an auxiliary result.

Lemma 4.2. Assume (4.1). Then the operator $P_{\gamma_{0} \mu}$ respects the maximum principle at $\left(t_{0}, x_{0}\right)$, that is, for any function $\phi$ such that $\phi\left(x_{0}\right) \geq \phi\left(x_{0}+\eta\right)$ for all $\eta \in \Lambda$, we have $P_{\gamma_{0} \mu} \phi\left(t_{0}, x_{0}\right) \leq 0$.

Proof. Since $P_{\gamma \mu} 1=0$ we may assume that $\phi\left(x_{0}\right)=0$. Then at $\left(t_{0}, x_{0}\right)$,

$$
P_{\gamma_{0} \mu} \phi=\left(v_{\gamma_{0}}^{-} \gamma_{0 i}-\mu v_{i}\right) \phi\left(x_{0}+h_{i} \ell_{i}\right)
$$

which is negative since $\phi\left(x_{0}+h_{i} \ell_{i}\right) \leq 0$ and $v_{\gamma_{0}}^{-} \gamma_{0 i}-\mu v_{i} \geq v_{\gamma_{0}}^{-}(\varepsilon-2 \mu) \geq 0(\mu \leq \varepsilon / 2)$. The lemma is proved.

We also need the following construction. Notice that, if $\left(t_{0}, x_{0}\right) \in Q$, there is a sequence $\alpha_{n} \in A$ such that at $\left(t_{0}, x_{0}\right)$,

$$
\begin{aligned}
& \lim _{n \rightarrow \infty}\left[r^{\alpha_{n}} \delta_{\tau}^{T} u+a_{k}^{\alpha_{n}} \Delta_{k} u+b_{k}^{\alpha_{n}} \delta_{k} u-c^{\alpha_{n}} u+g^{\alpha_{n}}\right] \\
& =\sup _{\alpha \in A}\left[r^{\alpha} \delta_{\tau}^{T} u+a_{k}^{\alpha} \Delta_{k} u+b_{k}^{\alpha} \delta_{k} u-c^{\alpha} u+g^{\alpha}\right]=0 .
\end{aligned}
$$

Since the number of possible values of $t$ for points in $Q$ is finite, and the functions $a_{k}^{\alpha}(t, x), b_{k}^{\alpha}(t, x), c^{\alpha}(t, x), f^{\alpha}(p, \psi, t, x)$ are uniformly continuous functions of $(p, \psi, x)$, there is a subsequence $\left\{n^{\prime}\right\} \subset\{1,2, \ldots\}$ and functions $\bar{r}, \bar{a}_{k}(t, x), \bar{b}_{k}(t, x)$, $\bar{c}(t, x), \bar{f}(p, \psi, t, x)$ such that they satisfy our assumptions changed in an obvious way and

$$
\begin{gathered}
\left(\bar{r}, \bar{a}_{k}(t, x), \bar{b}_{k}(t, x), \bar{c}(t, x), \bar{f}(p, \psi, t, x)\right) \\
=\lim _{n^{\prime} \rightarrow \infty}\left(r^{\alpha_{n^{\prime}}}(t), a_{k}^{\alpha_{n^{\prime}}}(t, x), b_{k}^{\alpha_{n^{\prime}}}(t, x), c^{\alpha_{n^{\prime}}}(t, x), f^{\alpha_{n^{\prime}}}(p, \psi, t, x)\right)
\end{gathered}
$$

on $Q$ for all $p, \psi$.

Obviously, for

$$
\bar{g}(t, x):=\bar{f}\left(\delta_{k} u(t, x), u(t, x), t, x\right)
$$

at $\left(t_{0}, x_{0}\right)$ we have

$$
\begin{gathered}
\bar{r} \delta_{\tau}^{T} u+\bar{a}_{k} \Delta_{k} u+\bar{b}_{k} \delta_{k} u-\bar{c} u+\bar{g}=0 \\
e^{-m \tau} \bar{r} \delta_{\tau}^{T} v+\bar{a}_{k} \Delta_{k} v+\bar{b}_{k} \delta_{k} v-\left(\bar{c}+\bar{r} c_{m}\right) v+\xi \bar{g}=0
\end{gathered}
$$

and, if $\left(t_{0}, x_{0}\right) \in Q_{1}^{o}$, then for any $i\left(= \pm 1, \ldots, \pm\left(d_{1}+1\right)\right.$, the shift operator $T$ is introduced in (3.1))

$$
T_{h_{i}, \ell_{i}}\left[e^{-m \tau} \bar{r} \delta_{\tau}^{T} v+\bar{a}_{k} \Delta_{k} v+\bar{b}_{k} \delta_{k} v-\left(\bar{c}+\bar{r} c_{m}\right) v+\xi \bar{g}\right] \leq 0,
$$

where here and below for simplicity of notation we drop $\left(t_{0}, x_{0}\right)$ in the arguments of functions we are dealing with.

Proof of Theorem 4.1. Set

$$
\bar{L}_{h}^{0}=\bar{a}_{k} \Delta_{k}+\bar{b}_{k} \delta_{k}, \quad \bar{L}_{h}=\bar{L}_{h}^{0}-\left(\bar{c}+\bar{r} c_{m}\right) .
$$

By Assumption 2.3, Lemma 3.1, and Lemma 3.2 (with $v_{\gamma}$ in place of $\phi$ )

$$
\begin{aligned}
0 & \geq \bar{L}_{h}^{0} V_{\gamma_{0} \mu}=2 v_{\gamma_{0}}^{-} \bar{L}_{h}^{0} v_{\gamma_{0}}^{-}+2 \mu v_{i} \bar{L}_{h}^{0} v_{i}+I_{1}+\mu I_{2} \\
& \geq-2 v_{\gamma_{0}}^{-} \bar{L}_{h}^{0} v_{\gamma_{0}}+2 \mu v_{i} \bar{L}_{h}^{0} v_{i}+I_{1}+\mu I_{2},
\end{aligned}
$$


where

$$
\begin{gathered}
I_{1}:=2 \bar{a}_{k}\left(\delta_{k} v_{\gamma_{0}}^{-}\right)^{2}+h \bar{b}_{k}\left(\delta_{k} v_{\gamma_{0}}^{-}\right)^{2}, \\
I_{2}:=2 \bar{a}_{k} \sum_{i}\left(\delta_{k} v_{i}\right)^{2}+h \bar{b}_{k} \sum_{i}\left(\delta_{k} v_{i}\right)^{2} .
\end{gathered}
$$

Since $2 \bar{a}_{k}+h \bar{b}_{k} \geq \bar{a}_{k}$ we conclude

$$
v_{\gamma_{0}}^{-} \bar{L}_{h}^{0} v_{\gamma_{0}}-\mu v_{i} \bar{L}_{h}^{0} v_{i} \geq(1 / 2) \mu \bar{a}_{k} \sum_{i}\left(\delta_{k} v_{i}\right)^{2} .
$$

On the other hand, by (4.2) and (4.3) and Lemma 4.2 at $\left(t_{0}, x_{0}\right)$,

$$
P_{\gamma_{0} \mu}\left[e^{-m \tau} \bar{r} \delta_{\tau}^{T} v+\bar{L}_{h} v+\xi \bar{g}\right] \leq 0 .
$$

Owing to (4.4) we obtain

$$
\begin{aligned}
& P_{\gamma_{0} \mu} \bar{L}_{h} v=v_{\gamma_{0}}^{-} \bar{L}_{h}^{0} v_{\gamma_{0}}-\mu v_{i} \bar{L}_{h}^{0} v_{i}+\left(\bar{c}+\bar{r} c_{m}\right) V_{\gamma_{0} \mu}+I_{3}+I_{4}+I_{5} \\
& \geq \lambda V_{\gamma_{0} \mu}+(1 / 2) \mu \bar{a}_{k} \sum_{i}\left(\delta_{k} v_{i}\right)^{2}+I_{3}+I_{4}+I_{5},
\end{aligned}
$$

where

$$
\begin{gathered}
I_{3}:=v_{\gamma_{0}}^{-}\left(P_{\gamma_{0}} \bar{a}_{k}\right) \Delta_{k} v+h_{i} v_{\gamma_{0}}^{-} \gamma_{0 i}\left(\delta_{i} \bar{a}_{k}\right) \Delta_{k} v_{i} \\
-\mu v_{i}\left(\delta_{i} \bar{a}_{k}\right) \Delta_{k} v-\mu h_{i} v_{i}\left(\delta_{i} \bar{a}_{k}\right) \Delta_{k} v_{i}, \\
I_{4}:=\left[v_{\gamma_{0}}^{-} \gamma_{0 i}-\mu v_{i}\right]\left(\delta_{i} \bar{b}_{k}\right) T_{h_{i}, \ell_{i}} v_{k}, \\
I_{5}:=\left[v_{\gamma_{0}}^{-} \gamma_{0 i}-\mu v_{i}\right]\left(\delta_{i} \bar{c}\right) T_{h_{i}, \ell_{i}} v .
\end{gathered}
$$

Upon observing that

$$
\Delta_{k}=-\delta_{k} \delta_{-k}, \quad h \Delta_{k}=\delta_{k}+\delta_{-k}, \quad h_{i} \leq h
$$

and, by assumption (4.1),

$$
h\left|\delta_{k} v_{-k}\right| \leq 2 \max _{\bar{Q}, i}\left|v_{i}\right| \leq 4 v_{\gamma_{0}}^{-}, \quad\left|h^{2} \Delta_{k} v_{i}\right| \leq 4 \max _{\bar{Q}}\left|v_{i}\right| \leq 8 v_{\gamma_{0}}^{-},
$$

we find

$$
\begin{gathered}
\left|I_{3}\right| \leq N v_{\gamma_{0}}^{-}\left(\sqrt{\bar{a}_{k}}+h\right)\left|\delta_{k} v_{-k}\right|+N v_{\gamma_{0}}^{-} \sum_{i}\left(\sqrt{\bar{a}_{k}}+h\right)\left|h \Delta_{k} v_{i}\right| \\
\leq N v_{\gamma_{0}}^{-} \sum_{i} \sqrt{\bar{a}_{k}}\left|\delta_{k} v_{i}\right|+N\left(v_{\gamma_{0}}^{-}\right)^{2} \leq N\left(v_{\gamma_{0}}^{-}\right)^{2}+(1 / 4) \mu \bar{a}_{k} \sum_{i}\left(\delta_{k} v_{i}\right)^{2} .
\end{gathered}
$$

This yields

$$
P_{\gamma_{0} \mu} \bar{L}_{h} v \geq \lambda V_{\gamma_{0} \mu}-N\left(v_{\gamma_{0}}^{-}\right)^{2}+(1 / 4) \mu \bar{a}_{k} \sum_{i}\left(\delta_{k} v_{i}\right)^{2}+I_{4}+I_{5} .
$$

Next, obviously,

$$
\left|I_{4}\right| \leq N\left(v_{\gamma_{0}}^{-}\right)^{2}, \quad\left|I_{5}\right| \leq N^{*} v_{\gamma_{0}}^{-} \max _{\bar{Q}}|v|
$$

Therefore, and since $\left(v_{\gamma_{0}}^{-}\right)^{2} \leq V_{\gamma_{0} \mu}$,

$$
P_{\gamma_{0} \mu} \bar{L}_{h} v \geq(\lambda-N) V_{\gamma_{0} \mu}-N^{*} \max _{Q}|v|^{2}+(1 / 4) \mu \bar{a}_{k} \sum_{i}\left(\delta_{k} v_{i}\right)^{2} .
$$

Now we deal with other terms in (4.5). Note that, since $\left(t_{0}+\tau_{T}\left(t_{0}\right), x_{0}\right) \in \bar{Q}$,

$$
0 \geq \delta_{\tau}^{T} V_{\gamma_{0} \mu}=2 v_{\gamma_{0}}^{-} \delta_{\tau}^{T} v_{\gamma_{0}}^{-}+2 \mu v_{i} \delta_{\tau}^{T} v_{i}+\tau\left(\delta_{\tau}^{T} v_{\gamma_{0}}^{-}\right)^{2}+\mu \tau \sum_{i}\left(\delta_{\tau}^{T} v_{i}\right)^{2},
$$


so that (cf. Lemma 3.2)

$$
0 \geq-v_{\gamma_{0}}^{-} \delta_{\tau}^{T} v_{\gamma_{0}}+\mu v_{i} \delta_{\tau}^{T} v_{i}=-P_{\gamma_{0} \mu} \delta_{\tau}^{T} v
$$

By recalling that $r^{\alpha} \geq 0$, we find from (4.6) and (4.5) that

$$
N^{*} \max _{\bar{Q}}|v|^{2} \geq(\lambda-N) V_{\gamma_{0} \mu}+\xi P_{\gamma_{0} \mu} \bar{g}+(1 / 4) \mu \bar{a}_{k} \sum_{i}\left(\delta_{k} v_{i}\right)^{2} .
$$

By Assumption 2.4.

$$
\left|\xi P_{\gamma_{0} \mu} \bar{g}\right| \leq N V_{\gamma_{0} \mu}^{1 / 2}\left(\sum_{i} \sqrt{\bar{a}_{k}}\left|\delta_{k} v_{i}\right|+V_{\gamma_{0} \mu}^{1 / 2}+N^{*} \xi\right) .
$$

It follows that the sum of the last two terms in (4.7) is greater than

$$
-N V_{\gamma_{0} \mu}-N^{*} \xi V_{\gamma_{0} \mu}^{1 / 2} \geq-N V_{\gamma_{0} \mu}-N^{*} \xi^{2} .
$$

Hence

$$
N^{*}\left(\max _{\bar{Q}}|v|^{2}+\xi^{2}\right) \geq\left(\lambda-N_{1}\right) V_{\gamma_{0} \mu},
$$

which for $\lambda-N_{1} \geq 1$ shows that on $\left.Q\right|_{0}$,

$$
\begin{gathered}
\left|\delta_{\eta, l} u\right|^{2}=\left|\delta_{\eta, l} v\right|^{2} \leq \mu^{-1} V_{\gamma_{0} \mu} \\
\leq N^{*}\left(\max _{\bar{Q}}|v|^{2}+\xi^{2}\right) \leq N^{*} \xi_{(+)}^{2}(T)\left(\max _{\bar{Q}}\left|\xi_{(-)} u\right|^{2}+1\right) .
\end{gathered}
$$

This implies (2.16) and the theorem is proved.

In light of this theorem to prove Theorem 2.5 we only need to show that in the case that the assumptions of Theorem 4.1 are not satisfied one can obtain the assertion of Theorem 2.5 differently. To do that we need two lemmas.

Lemma 4.3. Take a function $\phi$ on $\mathbb{R}^{d}$ and assume that

$$
\max _{i}\left|\delta_{i} \phi(x)\right| \leq \max _{i}\left|\delta_{i} \phi(0)\right|
$$

for $x \in \Lambda$. Then

$$
\max _{i}\left|\delta_{i} \phi(0)\right| \leq \max \left(\left(P_{\gamma} \phi(x)\right)^{-}: \gamma \in \Gamma, x \in\{0\} \cup \Lambda\right) .
$$

Proof. Take a $j$ such that

$$
\max _{i}\left|\delta_{i} \phi(0)\right|=\left|\delta_{j} \phi(0)\right|
$$

and first assume that

$$
\left|\delta_{j} \phi(0)\right|=-\delta_{j} \phi(0) .
$$

Then take $\gamma_{j}=\varepsilon^{-1}$ and $\gamma_{i}=\varepsilon$ for $i \neq j$. Since $-\delta_{j} \phi(0) \geq \delta_{i} \phi(0)$ and $-\delta_{j} \phi(0) \geq 0$, we have

$$
\begin{gathered}
P_{\gamma} \phi(0)=\varepsilon^{-1} \delta_{j} \phi(0)+\varepsilon \sum_{i \neq j} \delta_{i} \phi(0) \\
\leq\left(\varepsilon^{-1}-2 \varepsilon d_{1}\right) \delta_{j} \phi(0)=-\left(\varepsilon^{-1}-2 \varepsilon d_{1}\right) \max _{i}\left|\delta_{i} \phi(0)\right|
\end{gathered}
$$

and (4.8) follows since $\varepsilon^{-1}-2 \varepsilon d_{1}=1$.

If (4.9) is not satisfied, then

$$
\max _{i}\left|\delta_{i} \phi\left(h_{j} \ell_{j}\right)\right| \leq\left|\delta_{j} \phi(0)\right|=\delta_{j} \phi(0)=-\delta_{-j} \phi\left(h_{j} \ell_{j}\right),
$$


which combined with an obvious inequality between the extreme terms yields

$$
\begin{gathered}
\max _{i}\left|\delta_{i} \phi\left(h_{j} \ell_{j}\right)\right|=-\delta_{-j} \phi\left(h_{j} \ell_{j}\right)=\left|\delta_{-j} \phi\left(h_{j} \ell_{j}\right)\right| \\
=\left|\delta_{j} \phi(0)\right|=\max _{i}\left|\delta_{i} \phi(0)\right| .
\end{gathered}
$$

By the above argument applied to the point $h_{j} \ell_{j}$ in place of 0 ,

$$
\begin{aligned}
& \min _{\Gamma} P_{\gamma} \phi\left(h_{j} \ell_{j}\right) \leq\left(\varepsilon^{-1}-2 \varepsilon d_{1}\right) \delta_{-j} \phi\left(h_{j} \ell_{j}\right) \\
= & -\left(\varepsilon^{-1}-2 \varepsilon d_{1}\right) \max _{i}\left|\delta_{i} \phi(0)\right|=-\max _{i}\left|\delta_{i} \phi(0)\right| .
\end{aligned}
$$

The lemma is proved.

Lemma 4.4. Condition (4.1) is satisfied if $\left(t_{0}, x_{0}\right) \in Q_{1}^{o}$ and

$$
\max _{\bar{Q}, i} v_{i}^{2}=\max _{Q_{1}^{o}, i} v_{i}^{2} .
$$

Indeed if (4.1) does not hold, then

$$
\begin{gathered}
\max _{\Gamma \times \bar{Q}}\left[v_{\gamma}^{-}\right]^{2} \leq V_{\gamma_{0} \mu}\left(t_{0}, x_{0}\right) \\
<\left(2\left(d_{1}+1\right) \mu+1 / 4\right) \max _{\bar{Q}, i} v_{i}^{2}=\left(2\left(d_{1}+1\right) \mu+1 / 4\right) \max _{Q_{1}^{o}, i} v_{i}^{2},
\end{gathered}
$$

which contradicts Lemma 4.3 since $\mu \leq 1 /\left(4\left(d_{1}+1\right)\right)$.

Proof of Theorem 2.5. If $\left(t_{0}, x_{0}\right) \in \partial_{1} Q$, then in $\left.Q\right|_{0}$,

$$
\left|\delta_{\eta, l} u\right|=\left|\delta_{\eta, l} v\right| \leq \mu^{-1 / 2} V_{\gamma_{0} \mu}^{1 / 2} \leq \mu^{-1 / 2} V_{\gamma_{0} \mu}^{1 / 2}\left(t_{0}, x_{0}\right),
$$

where the last term is obviously less than the right-hand side of (2.16). Furthermore, if $\left(t_{0}, x_{0}\right) \in Q_{1}^{o}$ but (4.1) is violated, then by Lemma 4.4.

$$
\max _{\bar{Q}, i} v_{i}^{2}=\max _{\partial_{1} Q, i} v_{i}^{2}
$$

and one can use the above argument. Finally, in the remaining case, Theorem 4.1 is applicable. The theorem is proved.

Remark 4.5. As in [5] one can relax conditions on $\lambda$ by adding in its expression any large constant times $\min _{k} a_{k}^{\alpha}$ provided that $\Lambda=\Lambda_{0}$. This can be shown by considering maximum points of $V_{\gamma \mu}+\nu v^{2}$, where $\nu$ is a large constant. This would also allow us to relax the condition on $D_{\psi} f$ to $\left|D_{\psi} f\right| \leq K_{0}+K_{3} \min _{k} a_{k}^{\alpha}$.

\section{Proof of Theorem 2.9}

Our goal is to show how to choose an appropriate $C=C\left(d_{1}\right)$ in (2.18). Below in this section, by $N$ we denote generic constants depending only on $d_{1}, \delta, K_{1}, K_{2}, K_{3}$ but not $\omega$.

First of all, observe that $l$ does not enter either equation (2.9) or the statement of the theorem. It is involved, however, in the definition of $\partial_{1} Q$, making it "fatter". Because of that if we additionally assume that $l=0$, the result will be stronger. Therefore, we assume that $l=0$.

Set $m=0$ and introduce $\varepsilon, \Gamma, v_{\gamma}, v_{i}, V_{\gamma \mu}, W, P_{\gamma}, P_{\gamma \mu}$ as in the beginning of Section 4. However, since $v=u$, we also write $u_{\gamma}, u_{i}$, and $U_{\gamma \mu}$ instead of $v_{\gamma}, v_{i}$, and $V_{\gamma \mu}$, respectively. Since $l=0, u_{ \pm\left(d_{1}+1\right)}=v_{ \pm\left(d_{1}+1\right)}=0$ and now there is no need to allow $i$ to take the values $\pm\left(d_{1}+1\right)$. Therefore, we restrict $i$ to the range 
$\pm 1, \ldots, \pm d_{1}$. As everywhere in the article, index $k$ runs through $\pm 1, \ldots, \pm d_{1}$. This time we take

$$
8 \mu=d_{1}^{-1} \wedge \varepsilon
$$

Introduce $\kappa \geq 0$ as a solution of

$$
\max _{\Gamma \times \bar{Q}}\left[U_{\gamma \mu}+\kappa u^{2}\right]=4 \kappa K_{1}^{2} .
$$

Observe that if $\kappa=0$ is a solution of (5.1), then $u_{k} \equiv 0$ and the assertion of the theorem is trivial. Therefore, without losing generality we may assume that for $\kappa=$ 0 the left-hand side of (5.1) is strictly greater than its right-hand side. Furthermore, as a function of $\kappa$ the left-hand side is convex, increasing with Lipschitz constant not greater than $K_{1}^{2}$. It follows that (5.1) has a unique solution $\kappa>0$. After that we define $\left(\gamma_{0}, t_{0}, x_{0}\right)$ as a point in $\Gamma \times \bar{Q}$ at which the maximum in (5.1) is attained. For simplicity of notation we drop the arguments $\left(t_{0}, x_{0}\right)$ in what follows. We also use the abbreviated notation $\delta_{i}, \Delta_{k}$ introduced in Section 4

First we show that $\left[u_{\gamma_{0}}^{-}\right]^{2}$ is the main term in $U_{\gamma_{0} \mu}+\kappa u^{2}$.

Lemma 5.1. Assume that (4.10) holds. Then

$$
\begin{gathered}
{\left[u_{\gamma_{0}}^{-}\right]^{2} \geq 2 \kappa K_{1}^{2},} \\
2 d_{1} \varepsilon^{-1} \max _{i}\left|u_{i}\right| \geq u_{\gamma_{0}}^{-} \geq(1 / 2) \max _{\bar{Q}, i}\left|u_{i}\right| .
\end{gathered}
$$

In particular $u_{\gamma_{0}}<0$ and the operator $P_{\gamma_{0} \mu}$ respects the maximum principle at $\left(t_{0}, x_{0}\right)$.

Proof. First, notice that, owing to (4.10), Lemma 4.3, and the definitions of $\kappa$ and $\left(t_{0}, x_{0}\right)$ we have

$$
\begin{aligned}
& 4 \kappa K_{1}^{2}=\left[u_{\gamma_{0}}^{-}\right]^{2}+\mu \sum_{i} u_{i}^{2}+\kappa u^{2} \leq\left[u_{\gamma_{0}}^{-}\right]^{2}+2 d_{1} \mu \max _{\bar{Q}, i} u_{i}^{2}+\kappa K_{1}^{2} \\
& \leq\left[u_{\gamma_{0}}^{-}\right]^{2}+2 d_{1} \mu \max _{\Gamma \times \bar{Q}}\left[u_{\gamma}^{-}\right]^{2}+\kappa K_{1}^{2} \leq\left[u_{\gamma_{0}}^{-}\right]^{2}+2 d_{1} \mu 4 \kappa K_{1}^{2}+\kappa K_{1}^{2} .
\end{aligned}
$$

This implies (5.2) since $8 d_{1} \mu \leq 1$.

The first inequality in (5.3) is obvious. If the second one is wrong, then

$$
\begin{aligned}
\max _{\Gamma \times \bar{Q}}\left[u_{\gamma}^{-}\right]^{2} & \leq U_{\gamma_{0} \mu}+\kappa u^{2}<\left[2 d_{1} \mu+1 / 4\right] \max _{\bar{Q}, i} u_{i}^{2}+\kappa K_{1}^{2} \\
& \leq\left[2 d_{1} \mu+1 / 4\right] \max _{\Gamma \times \bar{Q}}\left[u_{\gamma}^{-}\right]^{2}+\kappa K_{1}^{2} \leq(1 / 2) \max _{\Gamma \times \bar{Q}}\left[u_{\gamma}^{-}\right]^{2}+\kappa K_{1}^{2}
\end{aligned}
$$

contrary to (5.2). The last assertion of the lemma follows from Lemma 4.2. The lemma is proved.

Next, if $\left(t_{0}, x_{0}\right) \in \partial_{1} Q$, then

$$
4 \kappa K_{1}^{2}=U_{\gamma_{0} \mu}+\kappa u^{2} \leq N^{\prime}+\kappa K_{1}^{2}, \quad 3 \kappa K_{1}^{2} \leq N^{\prime},
$$

and $u_{k}^{2}(t, x) \leq(4 / 3) \mu^{-1} N^{\prime}$ in $\bar{Q}$ according to (5.1). In this case the assertion of the theorem is true. Similarly, as in the proof of Theorem 2.5 we get the result if (4.10) is violated. This justifies the first two assumptions in the following set which we impose:

$$
\left(t_{0}, x_{0}\right) \in Q_{1}^{o}, \quad \max _{\bar{Q}, k} u_{k}^{2}=\max _{Q_{1}^{o}, k} u_{k}^{2},
$$




$$
4 d_{1} \varepsilon^{-1} W^{1 / 2} \geq \max _{Q_{1}^{o}, k}\left|u_{k}\right|, \quad W \geq K_{2}^{2}+8 K_{1} K_{3} \delta^{-1}+1 .
$$

The third relation in (5.4) follows from Lemma 5.1, and the last assumption in (5.4) restricts us to the only nontrivial case in light of (5.3).

Next again owing to the fact that the number of points in $Q$ is finite we can find some functions $\bar{r}(t), \bar{a}_{k}(t, x), \bar{c}(t, x)$, and $\bar{f}(p, \psi, r, t, x)$ satisfying Assumptions 2.1 and 2.5 and such that for

$$
\bar{g}(t, x):=\bar{f}\left(u_{k}(t, x), u(t, x), t, x\right)-\bar{c}(t, x) u(t, x), \quad \bar{L}_{h}:=\bar{a}_{k} \Delta_{k}
$$

we have (recall that the arguments $\left(t_{0}, x_{0}\right)$ are dropped)

$$
\bar{r} \delta_{\tau}^{T} u+\bar{L}_{h} u+\bar{g}=0, \quad T_{h, \ell_{i}}\left[\bar{r} \delta_{\tau}^{T} u+\bar{L}_{h} u+\bar{g}\right] \leq 0
$$

for any $i\left(= \pm 1, \ldots, \pm d_{1}\right)$.

Now, as before,

$$
\begin{aligned}
0 \geq \bar{L}_{h}\left[U_{\gamma_{0} \mu}+\kappa u^{2}\right] \geq & -2 u_{\gamma_{0}}^{-} \bar{L}_{h} u_{\gamma_{0}}+2 \mu u_{i} \bar{L}_{h} u_{i}+2 \kappa u \bar{L}_{h} u \\
& +2 \bar{a}_{k}\left[\left(\delta_{k} u_{\gamma_{0}}^{-}\right)^{2}+\mu \sum_{i}\left(\delta_{k} u_{i}\right)^{2}+\kappa\left(\delta_{k} u\right)^{2}\right] .
\end{aligned}
$$

Furthermore,

$$
\begin{gathered}
0 \geq \delta_{\tau}^{T}\left[U_{\gamma_{0} \mu}+\kappa u^{2}\right]=2 u_{\gamma_{0}}^{-} \delta_{\tau}^{T} u_{\gamma_{0}}^{-}+2 \mu u_{i} \delta_{\tau}^{T} u_{i}+2 \kappa u \delta_{\tau}^{T} u \\
+2 \tau\left[\left(\delta_{\tau}^{T} u_{\gamma_{0}}^{-}\right)^{2}+\mu \sum_{i}\left(\delta_{\tau}^{T} u_{i}\right)^{2}+\kappa\left(\delta_{\tau}^{T} u\right)^{2}\right] \\
\geq-2 u_{\gamma_{0}}^{-} \delta_{\tau}^{T} u_{\gamma_{0}}+2 \mu u_{i} \delta_{\tau}^{T} u_{i}+2 \kappa u \delta_{\tau}^{T} u .
\end{gathered}
$$

We multiply (5.7) by $\bar{r}$, add the result to (5.6), and use that $\bar{r} \geq 0, \bar{c} \geq-K_{3}$, $\bar{a}_{k} \geq \delta,|u| \leq K_{1}$, and

$$
u\left(\bar{r} \delta_{\tau}^{T} u+\bar{L}_{h} u\right)=-u \bar{g}=\bar{c} u^{2}-u(\bar{g}+\bar{c} u) \geq-K_{3} K_{1}-u(\bar{g}+\bar{c} u),
$$

where $|\bar{g}+\bar{c} u| \leq \omega W+K_{3}$ (recall (5.4)). Then we obtain

$$
\begin{gathered}
u\left(\bar{r} \delta_{\tau}^{T} u+\bar{L}_{h} u\right) \geq-\omega K_{1} W-2 K_{1} K_{3}, \\
u_{\gamma_{0}}^{-}\left[\bar{r} \delta_{\tau}^{T}+\bar{L}_{h}\right] u_{\gamma_{0}}-\mu u_{i}\left[\bar{r} \delta_{\tau}^{T}+\bar{L}_{h}\right] u_{i} \geq \delta \mu M+\kappa W\left(\delta-K_{1} \omega\right)-2 \kappa K_{1} K_{3},
\end{gathered}
$$

where

$$
M:=\sum_{i, k}\left(\delta_{k} u_{i}\right)^{2} .
$$

Since $C \geq 4$ in Assumption 2.6. we have $K_{1} \omega \leq \delta / 4$ which, along with (5.4), leads to

$$
\begin{gathered}
W\left(\delta-K_{1} \omega\right)-2 K_{1} K_{3} \geq(3 / 4) \delta W-2 K_{1} K_{3} \geq(\delta / 2) W \\
u_{\gamma_{0}}^{-}\left[\bar{r} \delta_{\tau}^{T}+\bar{L}_{h}\right] u_{\gamma_{0}}-\mu u_{i}\left[\bar{r} \delta_{\tau}^{T}+\bar{L}_{h}\right] u_{i} \geq \delta \mu M+(\delta / 2) \kappa W .
\end{gathered}
$$

On the other hand, owing to (5.4), (5.5), and Lemma 5.1,

$$
P_{\gamma_{0} \mu}\left[\bar{r} \delta_{\tau}^{T} u+\bar{L}_{h} u+\bar{g}\right] \leq 0 .
$$

Here due to (5.8),

$$
\begin{aligned}
P_{\gamma_{0} \mu}\left[\bar{r} \delta_{\tau}^{T} u+\bar{L}_{h} u\right] & =u_{\gamma_{0}}^{-}\left[\bar{r} \delta_{\tau}^{T}+\bar{L}_{h}\right] u_{\gamma_{0}}-\mu u_{i}\left[\bar{r} \delta_{\tau}^{T}+\bar{L}_{h}\right] u_{i}+I \\
& \geq \delta \mu M+(1 / 2) \delta \kappa W+I,
\end{aligned}
$$


where

$$
I:=\left[u_{\gamma_{0}}^{-} \gamma_{0 i}-\mu u_{i}\right]\left(\delta_{i} \bar{a}_{k}\right)\left[\Delta_{k} u+h \Delta_{k} u_{i}\right]
$$

Below by $C$ we denote generic constants depending only on $d_{1}$. It follows from the estimates

$$
\begin{gathered}
\left|\delta_{i} \bar{a}_{k}\right| \leq K_{3}+\omega\left|u_{i}\right|, \quad\left|h \Delta_{k} u_{i}\right|=\left|\delta_{k} u_{i}+\delta_{-k} u_{i}\right| \leq 2 M^{1 / 2}, \\
\left|\Delta_{k} u\right| \leq M^{1 / 2}, \quad\left|u_{\gamma_{0}}^{-} \gamma_{0 i}-\mu u_{i}\right| \leq C W^{1 / 2}
\end{gathered}
$$

that

$$
|I| \leq C W^{1 / 2} M^{1 / 2}\left(K_{3}+\omega W^{1 / 2}\right) \leq N W+(1 / 2) \delta \mu M+C \delta^{-1} \omega^{2} W^{2} .
$$

Hence,

$$
P_{\gamma_{0} \mu}\left[\bar{r} \delta_{\tau}^{T} u+\bar{L}_{h} u\right] \geq(1 / 2) \delta \mu M+(1 / 2) \delta \kappa W-N W-C \delta^{-1} \omega^{2} W^{2} .
$$

To estimate $P_{\gamma_{0} \mu} \bar{g}$ recall that $\bar{c} \geq-N, U_{\gamma_{0} \mu} \geq 0$ and observe that

$$
\begin{gathered}
-P_{\gamma_{0} \mu}(\bar{c} u)=-\bar{c} P_{\gamma_{0} \mu} u-\left[u_{\gamma_{0}}^{-} \gamma_{0 k}-\mu u_{k}\right]\left(T_{h, \ell_{k}} u\right) \delta_{k} \bar{c} \\
=\bar{c} U_{\gamma_{0} \mu}-\left[u_{\gamma_{0}}^{-} \gamma_{0 k}-\mu u_{k}\right]\left(T_{h, \ell_{k}} u\right) \delta_{k} \bar{c} \geq-N W-N W^{1 / 2} \geq-N W,
\end{gathered}
$$

where the last inequality follows from (5.4). Furthermore,

$$
h \delta_{i}(\bar{g}+\bar{c} u)=\bar{f}\left(T_{h, \ell_{i}} u_{k}, T_{h, \ell_{i}} u, t_{0}, x_{0}+h \ell_{i}\right)-\bar{f}\left(u_{k}, u, t_{0}, x_{0}\right) .
$$

Owing to Assumption 2.5. (5.4), and the mean value theorem (this is the place, where one cannot assume that (2.17) holds only for large $p$ )

$$
\begin{gathered}
\left|\delta_{i}(\bar{g}+\bar{c} u)\right| \leq C M^{1 / 2}\left(\omega W^{1 / 2}+K_{3}\right) \\
+C\left[W^{1 / 2}\left(\omega W+K_{3}\right)+\omega W^{3 / 2}+K_{3}\right] .
\end{gathered}
$$

Note that the coefficients of $\delta_{i}(\bar{g}+\bar{c} u)$ in $P_{\gamma_{0} \mu}(\bar{g}+\bar{c} u)$ are dominated by $W^{1 / 2}$ and for any $\rho>0$,

$$
\omega M^{1 / 2} W \leq \rho M+\rho^{-1} \omega^{2} W^{2}, \quad K_{3} M^{1 / 2} W^{1 / 2} \leq \rho M+\rho^{-1} N W .
$$

Therefore,

$$
\begin{gathered}
\left|P_{\gamma_{0} \mu}(\bar{g}+\bar{c} u)\right| \leq(1 / 2) \delta \mu M+C\left[\delta^{-1} \omega^{2}+\omega\right] W^{2}+N W, \\
P_{\gamma_{0} \mu} \bar{g} \geq-(1 / 2) \delta \mu M-C\left[\delta^{-1} \omega^{2}+\omega\right] W^{2}-N W
\end{gathered}
$$

and (5.9) and (5.10) yield

$$
(1 / 2) \delta \kappa W \leq C\left[\delta^{-1} \omega^{2}+\omega\right] W^{2}+N W,
$$

$$
2 \delta \kappa \leq C_{1}\left[\delta^{-1} \omega^{2}+\omega\right] W+N_{1} .
$$

Since $W \leq \mu^{-1} 4 K_{1}^{2} \kappa$ (see (5.1) ), we see that if $C$ in Assumption 2.6 is such that

$$
C \geq 4 \mu^{-1} C_{1}
$$

then (recall that $C \geq 4$ and $\omega K_{1} \leq \delta$ )

$$
C_{1}\left[\delta^{-1} \omega^{2}+\omega\right] W \leq C K_{1}^{2}\left[\delta^{-1} \omega^{2}+\omega\right] \kappa \leq C\left[K_{1} \omega+K_{1}^{2} \omega\right] \kappa \leq \delta \kappa .
$$

In this case (5.11) allows us to conclude that $\kappa \leq \delta^{-1} N_{1}$ and we get the assertion of the theorem from (5.1). The theorem is proved. 


\section{Conditional EStimates of The SECOND-ORDER DifFEREnCES}

In this section we suppose that all the assumptions of Theorem 2.12 are satisfied apart from Assumptions 2.8 and 2.9. The notation in this section is somewhat different from Sections 4 and 5 . Of course, we use our basic notation from Section 2. for instance, $\xi$ and $\lambda$ are defined in (2.14).

For $\varepsilon \in(0,1]$ set (observe that now $\gamma_{k}=\gamma_{-k}$ )

$$
\Gamma(\varepsilon)=\left\{\gamma=\left(\gamma_{k}: k= \pm 1, \ldots, \pm d_{1}\right): \gamma_{k}=\gamma_{-k}, \varepsilon \leq \gamma_{k} \leq \varepsilon^{-1}, \forall k\right\}
$$

Fix a constant $\mu=\mu\left(d_{1}, \varepsilon\right)>0$ such that

$$
16 d_{1}^{2} \mu \leq \varepsilon^{2} .
$$

In this section the indices $i, j, k, p, q$ run through $\left\{ \pm 1, \ldots, \pm d_{1}\right\}$. The main result of this section is the following.

Theorem 6.1. Assume that

$$
3 \mu \max _{Q} \sum_{i, j}\left[\left(\xi \delta_{h, \ell_{j}} \delta_{h, \ell_{i}} u\right)^{-}\right]^{2} \leq \max _{\Gamma(\varepsilon) \times Q}\left[\left(\xi \sum_{i} \gamma_{i} \Delta_{h, \ell_{i}} u\right)^{-}\right]^{2} .
$$

Then there exists a constant $N=N\left(\varepsilon, \mu, d_{1}, K_{0}\right)$ such that if $\lambda \geq N$, then in $\left.Q\right|_{0}$ for $i, j= \pm 1, \ldots, \pm d_{1}$ we have

$$
\begin{gathered}
\left|\delta_{h, \ell_{j}} \delta_{h, \ell_{i}} u\right| \leq N^{*} e^{m^{+}(T+\tau)}\left(1+\max _{\partial_{2} Q, p, q}\left|\xi_{(-)} \delta_{h, \ell_{p}} \delta_{h, \ell_{q}} u\right|\right. \\
\left.+\max _{\bar{Q}}\left|\xi_{(-)} u\right|+\max _{\bar{Q}, p}\left|\xi_{(-)} \delta_{h, \ell_{p}} u\right|\right),
\end{gathered}
$$

where $N^{*}=N^{*}\left(h_{0}, \varepsilon, \mu, d_{1}, K_{0}, K_{3}\right)$.

Below in this section, by $N$ and $N^{*}$ we denote generic constants of the same type as in the theorem. As before, we use the abbreviated notation

$$
\Delta_{i}=\Delta_{h, \ell_{i}}, \quad \delta_{i}=\delta_{h, \ell_{i}} .
$$

Introduce $v=\xi u$ as in Section 4 and fix a constant $\nu \geq 1$. Set

$$
\begin{gathered}
P_{\gamma}=\gamma_{i} \Delta_{i}, \quad v_{\gamma}=P_{\gamma} v, \quad v_{i}=\delta_{i} v, \quad v_{i j}=\delta_{j} \delta_{i} v \\
P_{\gamma \mu \nu} \phi=v_{\gamma}^{-} P_{\gamma} \phi+\mu v_{i j}^{-} \delta_{j} \delta_{i} \phi-\nu v_{i} \delta_{i} \phi \\
W_{1}=\sum_{i} v_{i}^{2}, \quad W_{2}=\sum_{i, j}\left[v_{i j}^{-}\right]^{2}, \quad V_{\gamma \mu \nu}=\left[v_{\gamma}^{-}\right]^{2}+\mu W_{2}+\nu W_{1} .
\end{gathered}
$$

Observe that this time again $P_{\gamma \mu \nu} v=-V_{\gamma \mu \nu}$ and also note that (6.1) is equivalent to the following:

$$
3 \mu \max _{Q} W_{2} \leq \max _{\Gamma(\varepsilon) \times Q}\left(v_{\gamma}^{-}\right)^{2}
$$

We introduce $\left(\gamma_{0}, t_{0}, x_{0}\right)$ as a point in $\Gamma(\varepsilon) \times \bar{Q}$ maximizing $V_{\gamma \mu \nu}$ and first prove a few auxiliary results. Below, as usual, we drop the arguments $\left(t_{0}, x_{0}\right)$.

Lemma 6.2. (i) For $(t, x) \in Q_{1}^{o}$ and any $i, j$,

$$
\left|v_{i j}(t, x)\right| \leq \max _{Q} W_{2}^{1 / 2} .
$$

(ii) If (6.3) holds and $\left(t_{0}, x_{0}\right) \in Q$ and

$$
\nu \max _{Q} W_{1} \leq \mu \max _{Q} W_{2},
$$


then at $\left(t_{0}, x_{0}\right)$,

$$
\mu \max _{Q} W_{2} \leq\left[v_{\gamma_{0}}^{-}\right]^{2}, \quad \nu \max _{Q} W_{1} \leq\left[v_{\gamma_{0}}^{-}\right]^{2} .
$$

Furthermore, if additionally,

$$
h \sqrt{\nu} \leq \varepsilon
$$

then the operator $P_{\gamma_{0} \mu \nu}$ respects the maximum principle at $\left(t_{0}, x_{0}\right)$ relative to $\Lambda_{0}+$ $\Lambda_{0}$, that is, for any function $\phi$ such that $\phi\left(x_{0}\right) \geq \phi\left(x_{0}+\eta\right)$ for all $\eta \in \Lambda_{0}+\Lambda_{0}$, we have $P_{\gamma_{0} \mu \nu} \phi\left(t_{0}, x_{0}\right) \leq 0$.

Proof. (i) Obviously $v_{-i, j}^{-} \leq W_{2}^{1 / 2}$ on $Q$. Since (no summation in $i$ ) $T_{h, \ell_{i}} v_{-i, j}^{-}=v_{i j}^{+}$, we get $v_{i j}^{+}(t, x) \leq W_{2}^{1 / 2}\left(t, x+h \ell_{i}\right)$. This proves (i).

(ii) The second estimate in (6.6) follows from the first one and (6.5). Assuming that the first estimate in (6.6) does not hold, we obtain at $\left(t_{0}, x_{0}\right)$,

$$
\begin{gathered}
V_{\gamma_{0} \mu \nu}<2 \mu \max _{Q} W_{2}+\nu \max _{Q} W_{1} \leq 3 \mu \max _{Q} W_{2}, \\
\max _{\Gamma(\varepsilon) \times Q}\left[v_{\gamma}^{-}\right]^{2} \leq V_{\gamma_{0} \mu \nu}<3 \mu \max _{Q} W_{2},
\end{gathered}
$$

contrary to (6.3). This proves (6.6).

To prove the last assertion of the lemma we take a function with described properties and without loss of generality assume that $\phi\left(x_{0}\right)=0$. We also note that

$$
\begin{gathered}
h^{2} \delta_{i} \delta_{j} \phi\left(x_{0}\right)=\phi\left(x_{0}+h \ell_{i}+h \ell_{j}\right)-\phi\left(x_{0}+h \ell_{i}\right)-\phi\left(x_{0}+h \ell_{j}\right) \\
+\phi\left(x_{0}\right) \leq-\phi\left(x_{0}+h \ell_{i}\right)-\phi\left(x_{0}+h \ell_{j}\right)
\end{gathered}
$$

and $\Delta_{i} \phi\left(x_{0}\right) \leq 0$. Therefore, as usual dropping the arguments $\left(t_{0}, x_{0}\right)$ in $v_{\ldots}$, we infer from (6.6) that

$$
\begin{gathered}
h^{2} P_{\gamma_{0} \mu \nu} \phi\left(t_{0}, x_{0}\right) \leq v_{\gamma_{0}}^{-} \varepsilon \sum_{i}\left(\phi\left(x_{0}+h \ell_{i}\right)+\phi\left(x_{0}-h \ell_{i}\right)\right) \\
-\mu v_{i j}^{-}\left(\phi\left(x_{0}+h \ell_{i}\right)+\phi\left(x_{0}+h \ell_{j}\right)\right)-h \nu v_{i} \phi\left(x_{0}+h \ell_{i}\right) \\
\leq v_{\gamma_{0}}^{-}\left[\varepsilon \sum_{i}\left(\phi\left(x_{0}+h \ell_{i}\right)+\phi\left(x_{0}-h \ell_{i}\right)\right)\right. \\
\left.-\sqrt{\mu} \sum_{i, j}\left(\phi\left(x_{0}+h \ell_{i}\right)+\phi\left(x_{0}+h \ell_{j}\right)\right)-h \sqrt{\nu} \sum_{i} \phi\left(x_{0}+h \ell_{i}\right)\right] \\
=v_{\gamma_{0}}^{-} \sum_{i} \phi\left(x_{0}+h \ell_{i}\right)\left[2 \varepsilon-2 d_{1} \sqrt{\mu}-h \sqrt{\nu}\right] .
\end{gathered}
$$

The last expression is less than zero in light of the fact that $4 d_{1} \sqrt{\mu} \leq \varepsilon$ and $h \sqrt{\nu} \leq \varepsilon$. The lemma is proved.

Remark 6.3. This lemma can be generalized to the case when the $\ell_{k}$ 's come with different $h_{k}$ 's, but the $h_{k}$ 's should be comparable. This is the reason why in Theorem 2.12 we do not include $h_{d_{1}+1}$ and $\ell_{d_{1}+1}$.

Set

$$
\begin{gathered}
Z_{k}:=v_{\gamma_{0}}^{-} \Delta_{k} v_{\gamma_{0}}+\mu v_{i j}^{-} \Delta_{k} v_{i j}-\nu v_{i} \Delta_{k} v_{i} \\
z_{k}:=v_{\gamma_{0}}^{-} \delta_{k} v_{\gamma_{0}}+\mu v_{i j}^{-} \delta_{k} v_{i j}-\nu v_{i} v_{k i} \\
R_{k}^{\gamma}:=\left[\delta_{k} v_{\gamma_{0}}^{-}\right]^{2}, \quad R_{k}^{\mu}:=\sum_{i, j}\left[\delta_{k} v_{i j}^{-}\right]^{2}, \quad R_{k}^{\nu}:=\sum_{i} v_{k i}^{2} .
\end{gathered}
$$


These objects evaluated at $\left(t_{0}, x_{0}\right)$ will be extensively used below in this section.

Lemma 6.4. If $\left(t_{0}, x_{0}\right) \in Q_{1}^{o}$, then at $\left(t_{0}, x_{0}\right)$ we have for any $k$,

$$
\begin{aligned}
2 Z_{k} & \geq-2 v_{\gamma_{0}}^{-} \Delta_{k} v_{\gamma_{0}}^{-}-2 \mu v_{i j}^{-} \Delta_{k} v_{i j}^{-}-2 \nu v_{i} \Delta_{k} v_{i} \\
& \geq R_{k}^{\gamma}+R_{-k}^{\gamma}+\mu R_{k}^{\mu}+\mu R_{-k}^{\mu}+\nu R_{k}^{\nu}+\nu R_{-k}^{\nu} \geq 0 .
\end{aligned}
$$

Furthermore, for any $\alpha \in A$ and $h \leq h_{0} / 2$,

$$
4\left(a_{k}^{\alpha} Z_{k}+b_{k}^{\alpha} z_{k}\right) \geq 2 a_{k}^{\alpha} Z_{k}+a_{k}^{\alpha}\left(R_{k}^{\gamma}+\mu R_{k}^{\mu}+\nu R_{k}^{\nu}\right) .
$$

Proof. The first inequality in (6.7) follows from Lemma 3.2. To prove the second one it suffices to observe that

$$
\begin{aligned}
0 \geq & \Delta_{k} V_{\gamma_{0} \mu \nu}=2 v_{\gamma_{0}}^{-} \Delta_{k} v_{\gamma_{0}}^{-}+2 \mu v_{i j}^{-} \Delta_{k} v_{i j}^{-}+2 \nu v_{i} \Delta_{k} v_{i} \\
& +R_{k}^{\gamma}+R_{-k}^{\gamma}+\mu R_{k}^{\mu}+\mu R_{-k}^{\mu}+\nu R_{k}^{\nu}+\nu R_{-k}^{\nu} .
\end{aligned}
$$

Next, using that $h \leq h_{0} / 2$, by (6.7) and Assumption 2.3 we get

$$
\begin{gathered}
0 \geq\left(a_{k}^{\alpha} \Delta_{k}+2 b_{k}^{\alpha} \delta_{k}\right) V_{\gamma_{0} \mu \nu}=2 v_{\gamma_{0}}^{-}\left[a_{k}^{\alpha} \Delta_{k}+2 b_{k}^{\alpha} \delta_{k}\right] v_{\gamma_{0}}^{-} \\
+2 \mu v_{i j}^{-}\left[a_{k}^{\alpha} \Delta_{k}+2 b_{k}^{\alpha} \delta_{k}\right] v_{i j}^{-}+2 \nu v_{i}\left[a_{k}^{\alpha} \Delta_{k}+2 b_{k}^{\alpha} \delta_{k}\right] v_{i} \\
+2 a_{k}^{\alpha}\left[R_{k}^{\gamma}+\mu R_{k}^{\mu}+\nu R_{k}^{\nu}\right]+2 h b_{k}^{\alpha}\left[R_{k}^{\gamma}+\mu R_{k}^{\mu}+\nu R_{k}^{\nu}\right] \\
\geq-2 v_{\gamma_{0}}^{-}\left[a_{k}^{\alpha} \Delta_{k}+2 b_{k}^{\alpha} \delta_{k}\right] v_{\gamma_{0}} \\
-2 \mu v_{i j}^{-}\left[a_{k}^{\alpha} \Delta_{k}+2 b_{k}^{\alpha} \delta_{k}\right] v_{i j}+2 \nu v_{i}\left[a_{k}^{\alpha} \Delta_{k}+2 b_{k}^{\alpha} \delta_{k}\right] v_{i} \\
+2 a_{k}^{\alpha}\left[R_{k}^{\gamma}+\mu R_{k}^{\mu}+\nu R_{k}^{\nu}\right]+2 h b_{k}^{\alpha}\left[R_{k}^{\gamma}+\mu R_{k}^{\mu}+\nu R_{k}^{\nu}\right] \\
\geq-2 a_{k}^{\alpha} Z_{k}-4 b_{k}^{\alpha} z_{k}+a_{k}^{\alpha}\left[R_{k}^{\gamma}+\mu R_{k}^{\mu}+\nu R_{k}^{\nu}\right]
\end{gathered}
$$

and (6.8) follows. The lemma is proved.

In the following lemma we do the most important step in the proof of Theorem 6.1, Set

$$
\bar{W}_{1}:=\max _{Q} W_{1} .
$$

Lemma 6.5. Under the assumptions of Theorem 6.1 there are constants $N, N^{*}$,

$$
\nu=\nu^{*}\left(h_{0}, \varepsilon, \mu, d_{1}, K_{0}, K_{3}\right) \geq 1, \quad h^{*}=h^{*}\left(h_{0}, \varepsilon, \mu, d_{1}, K_{0}, K_{3}\right)>0
$$

such that $h^{*} \leq h_{0} / 2$ and, if condition (6.5) is satisfied and $h \in\left(0, h^{*}\right]$ and $\left(t_{0}, x_{0}\right) \in$ $Q_{2}^{o}$, then at $\left(t_{0}, x_{0}\right)$ for any $\alpha \in A$ we have

$$
J:=P_{\gamma_{0} \mu \nu}\left(a_{k}^{\alpha} \Delta_{k} v+b_{k}^{\alpha} \delta_{k} v\right) \geq-N\left[v_{\gamma_{0}}^{-}\right]^{2}-N^{*} \bar{W}_{1} .
$$

Proof. We fix an $\alpha \in A$ and drop the superscript $\alpha$ for convenience. By (3.2) (no summation in $k, i, j)$

$$
\begin{gathered}
\delta_{j} \delta_{i}\left(b_{k} v_{k}\right)=b_{k} \delta_{k} v_{i j}+\left(\delta_{j} b_{k}\right) v_{k i}+\left(\delta_{i} b_{k}\right) v_{k j} \\
+h\left[\left(\delta_{i}+\delta_{j}\right) b_{k}\right] \delta_{k} v_{i j}+\left(\delta_{j} \delta_{i} b_{k}\right) T_{h, \ell_{i}+\ell_{j}} v_{k} .
\end{gathered}
$$

Also by using (3.2) and the formulas $a_{k}=a_{-k}$ and $h \Delta_{k}=\delta_{k}+\delta_{-k}$ and summing with respect to $k$ (but not in $i, j$ ) we get

$$
\begin{gathered}
\delta_{j} \delta_{i}\left(a_{k} \Delta_{k} v\right)=a_{k} \Delta_{k} v_{i j}+\left(\delta_{j} a_{k}\right) \Delta_{k} v_{i}+\left(\delta_{i} a_{k}\right) \Delta_{k} v_{j} \\
+2\left[\left(\delta_{j}+\delta_{i}\right) a_{k}\right] \delta_{k} v_{i j}+\left(\delta_{j} \delta_{i} a_{k}\right) T_{h, \ell_{j}+\ell_{i}} \Delta_{k} v .
\end{gathered}
$$

While applying this formula to $\Delta_{i}$ it is also useful to observe that

$$
\left[\left(\delta_{-i}+\delta_{i}\right) a_{k}\right] \delta_{k} v_{i,-i}=-h\left(\Delta_{i} a_{k}\right) \Delta_{i} \delta_{k} v=-\left(\Delta_{i} a_{k}\right)\left(v_{k i}+v_{k,-i}\right) .
$$


Hence (recall that $\gamma_{0 i}=\gamma_{0,-i}$ and $\ell_{i}=-\ell_{-i}$ ),

$$
\begin{gathered}
P_{\gamma_{0}}\left(a_{k} \Delta_{k} v+b_{k} \delta_{k} v\right)=-\gamma_{0 i} \delta_{-i} \delta_{i}\left(a_{k} \Delta_{k} v+b_{k} \delta_{k} v\right) \\
=\left(a_{k} \Delta_{k}+b_{k} \delta_{k}\right) v_{\gamma_{0}}-2 \gamma_{0 i}\left(\delta_{-i} a_{k}\right) \Delta_{k} v_{i}+4 \gamma_{0 i}\left(\Delta_{i} a_{k}\right) v_{k i} \\
+\gamma_{0 i}\left(\Delta_{i} a_{k}\right) \Delta_{k} v+2 \gamma_{0 i}\left(\delta_{i} b_{k}\right) v_{k i}+\gamma_{0 i}\left(\Delta_{i} b_{k}\right) v_{k} .
\end{gathered}
$$

Also, everywhere,

$$
\begin{gathered}
v_{i j}^{-} \delta_{j} \delta_{i}\left(a_{k} \Delta_{k}+b_{k} \delta_{k}\right) v=v_{i j}^{-}\left(a_{k} \Delta_{k}+b_{k} \delta_{k}\right) v_{i j}+2 v_{i j}^{-}\left(\delta_{j} a_{k}\right) \Delta_{k} v_{i} \\
+h v_{i j}^{-}\left[\left(\delta_{j}+\delta_{i}\right) a_{k}\right] \Delta_{k} v_{i j}+v_{i j}^{-}\left(\delta_{j} \delta_{i} a_{k}\right) T_{h, \ell_{j}+\ell_{i}} \Delta_{k} v \\
+2 v_{i j}^{-}\left(\delta_{j} b_{k}\right) v_{k i}+2 h v_{i j}^{-}\left(\delta_{i} b_{k}\right) \delta_{k} v_{i j}+v_{i j}^{-}\left(\delta_{j} \delta_{i} b_{k}\right) T_{h, \ell_{i}+\ell_{j}} v_{k} .
\end{gathered}
$$

Therefore at $\left(t_{0}, x_{0}\right)$ we have

$$
J=a_{k} Z_{k}+b_{k} z_{k}+I_{1}+\ldots+I_{4},
$$

where

$$
\begin{gathered}
I_{1}=-2 \gamma_{0 i} v_{\gamma_{0}}^{-}\left(\delta_{-i} a_{k}\right) \Delta_{k} v_{i}+2 \mu v_{i j}^{-}\left(\delta_{j} a_{k}\right) \Delta_{k} v_{i}, \\
I_{2}=\mu h v_{i j}^{-}\left[\left(\delta_{j}+\delta_{i}\right) a_{k}\right] \Delta_{k} v_{i j}, \\
I_{3}=v_{\gamma_{0}}^{-}\left[4 \gamma_{0 i}\left(\Delta_{i} a_{k}\right) v_{k i}+\gamma_{0 i}\left(\Delta_{i} a_{k}\right) \Delta_{k} v\right] \\
+\mu v_{i j}^{-}\left(\delta_{j} \delta_{i} a_{k}\right) T_{h, \ell_{j}+\ell_{i}} \Delta_{k} v, \\
I_{4}=2 v_{\gamma_{0}}^{-} \gamma_{0 i}\left(\delta_{i} b_{k}\right) v_{k i}+v_{\gamma_{0}}^{-} \gamma_{0 i}\left(\Delta_{i} b_{k}\right) v_{k} \\
+2 \mu v_{i j}^{-}\left(\delta_{j} b_{k}\right) v_{k i}+2 \mu h v_{i j}^{-}\left(\delta_{i} b_{k}\right) \delta_{k} v_{i j}+\mu v_{i j}^{-}\left(\delta_{j} \delta_{i} b_{k}\right) T_{h, \ell_{i}+\ell_{j}} v_{k} \\
-\nu v_{i}\left(\delta_{i} a_{k}\right)\left(\Delta_{k} v+2 v_{k i}\right)-\nu v_{i}\left(\delta_{i} b_{k}\right) T_{h, \ell_{i}} v_{k} .
\end{gathered}
$$

For $h \leq h_{0} / 2$ it follows by Lemma 6.4 that

$$
4 J \geq 2 a_{k} Z_{k}+a_{k}\left(R_{k}^{\gamma}+\mu R_{k}^{\mu}+\nu R_{k}^{\nu}\right)+4 I_{1}+\ldots+4 I_{4} .
$$

Estimating $I_{1}$. Note that owing to (6.6),

$$
\left|4 I_{1}\right| \leq N v_{\gamma_{0}}^{-}\left(\sqrt{a_{k}}+h\right) \sum_{i}\left|\Delta_{k} v_{i}\right|
$$

and by (3.3),

$$
\begin{gathered}
N v_{\gamma_{0}}^{-} \sqrt{a_{k}} \sum_{i}\left|\Delta_{k} v_{i}\right| \leq N v_{\gamma_{0}}^{-} \sqrt{a_{k}} \sum_{i}\left[\left|\delta_{-k} v_{k i}^{-}\right|+\left|\delta_{k} v_{-k, i}^{-}\right|\right] \\
\leq N\left(v_{\gamma_{0}}^{-}\right)^{2}+(1 / 3) \mu a_{k} R_{k}^{\mu} .
\end{gathered}
$$

Furthermore, by the formula $h \Delta_{k}=\delta_{k}+\delta_{h, \ell_{-k}}$ and Lemma 6.2 we obtain

$$
v_{\gamma_{0}}^{-} h \sum_{k, i}\left|\Delta_{k} v_{i}\right| \leq 2 v_{\gamma_{0}}^{-} \sum_{k, i}\left|v_{k i}\right| \leq N v_{\gamma_{0}}^{-} \max _{Q} W_{2}^{1 / 2} \leq N\left(v_{\gamma_{0}}^{-}\right)^{2} .
$$

Thus,

$$
\left|4 I_{1}\right| \leq N\left(v_{\gamma_{0}}^{-}\right)^{2}+(1 / 3) \mu a_{k} R_{k}^{\mu} .
$$

Estimating $I_{2}$. Observe that

$$
\left|4 I_{2}\right| \leq N h v_{i j}^{-}\left(\sqrt{a_{k}}+h\right)\left|\Delta_{k} v_{i j}\right| \leq I_{21}+I_{22},
$$

where (see Lemma 6.2 and recall that $\left(t_{0}, x_{0}\right) \in Q_{2}^{0}$ )

$$
I_{21}=N \sum_{k} h^{2} v_{i j}^{-}\left|\Delta_{k} v_{i j}\right|=N \sum_{k} v_{i j}^{-}\left|\left(T_{h, \ell_{k}}-2+T_{h, \ell_{-k}}\right) v_{i j}\right| \leq N\left(v_{\gamma_{0}}^{-}\right)^{2} \text {, }
$$


and by the formula $|\theta|=\theta+2 \theta^{-}$,

$$
\begin{gathered}
I_{22}=N h v_{i j}^{-} \sqrt{a_{k}}\left|\Delta_{k} v_{i j}\right|=N h v_{i j}^{-} \sqrt{a_{k}} \Delta_{k} v_{i j} \\
+2 N h v_{i j}^{-} \sqrt{a_{k}}\left(\Delta_{k} v_{i j}\right)^{-}=N h \sqrt{a_{k}} Z_{k}+N h v_{i j}^{-} \sqrt{a_{k}}\left(\Delta_{k} v_{i j}\right)^{-}+I_{23},
\end{gathered}
$$

where

$$
I_{23}=-N h \sqrt{a_{k}}\left(v_{\gamma_{0}}^{-} \Delta_{k} v_{\gamma_{0}}-\nu v_{i} \Delta_{k} v_{i}\right) .
$$

Furthermore, by Lemma 3.2 ,

$$
N h v_{i j}^{-} \sqrt{a_{k}}\left(\Delta_{k} v_{i j}\right)^{-} \leq N h \sqrt{a_{k}} v_{i j}^{-}\left|\Delta_{k} v_{i j}^{-}\right|=N \sqrt{a_{k}} v_{i j}^{-}\left|\left(\delta_{k}+\delta_{-k}\right) v_{i j}^{-}\right|,
$$

which is majorized by the right-hand side of (6.11).

To estimate $I_{23}$ we use Lemma 3.2 to get

$$
\begin{aligned}
-N h \sqrt{a_{k}} v_{\gamma_{0}}^{-} \Delta_{k} v_{\gamma_{0}} & =-N \sqrt{a_{k}} v_{\gamma_{0}}^{-} \delta_{k} v_{\gamma_{0}} \leq N \sqrt{a_{k}} v_{\gamma_{0}}^{-} \delta_{k} v_{\gamma_{0}}^{-} \\
& \leq N\left(v_{\gamma_{0}}^{-}\right)^{2}+(1 / 3) a_{k} R_{k}^{\gamma} .
\end{aligned}
$$

Furthermore, by assumption (6.5),

$$
N h \nu v_{i} \sqrt{a_{k}} \Delta_{k} v_{i}=N \nu v_{i} \sqrt{a_{k}} v_{k i} \leq N\left(v_{\gamma_{0}}^{-}\right)^{2}+(1 / 3) \nu a_{k} R_{k}^{\nu} .
$$

It follows that

$$
I_{23} \leq N\left(v_{\gamma_{0}}^{-}\right)^{2}+(1 / 3) a_{k}\left[\delta_{k} v_{\gamma_{0}}^{-}\right]^{2}+(1 / 3) \nu a_{k} R_{k}^{\nu}
$$

Hence

$$
\left|4 I_{2}\right| \leq N\left(v_{\gamma_{0}}^{-}\right)^{2}+N h \sqrt{a_{k}} Z_{k}+(1 / 3) a_{k}\left(R_{k}^{\gamma}+\mu R_{k}^{\mu}+\nu R_{k}^{\nu}\right)
$$

Estimating $I_{3}$. We use the following result of simple computations:

$$
T_{h, \ell_{j}+\ell_{i}} \Delta_{k} v=-v_{k,-k}+v_{k j}+v_{-k, j}+v_{k i}+v_{-k, i}+h^{2} \Delta_{k} v_{i j} .
$$

This shows new terms entering $I_{3}$. All of them, apart from the last one, are similar to the ones which are written explicitly in the definition of $I_{3}$, and we show how to estimate only one of them. By Assumption 2.7 and Lemma 6.2 we have

$$
\left|\mu v_{i j}^{-}\left(\delta_{j} \delta_{i} a_{k}\right) v_{k,-k}\right| \leq N\left(v_{\gamma_{0}}^{-}\right)^{2}+N^{*}\left|v_{\gamma_{0}}^{-}\right| \sum_{k, i} \sqrt{a_{k}}\left|v_{k i}\right| \leq N\left(v_{\gamma_{0}}^{-}\right)^{2}+N^{*} a_{k} R_{k}^{\nu} .
$$

To estimate the remaining term in $I_{3}$ we proceed as in estimating $I_{2}$. We have

$$
\begin{gathered}
\mu h^{2} v_{i j}^{-}\left(\delta_{j} \delta_{i} a_{k}\right) \Delta_{k} v_{i j} \\
\leq N h^{2} v_{i j}^{-}\left(N+N^{*} \sqrt{a_{k}}\right)\left|\Delta_{k} v_{i j}\right|=I_{31}+I_{32} .
\end{gathered}
$$

Here by Lemma 6.2 and because $\left(t_{0}, x_{0}\right) \in Q_{2}^{o}$,

$$
I_{31}=N h^{2} v_{i j}^{-}\left|\Delta_{k} v_{i j}\right|=N v_{i j}^{-}\left|\left(T_{h, \ell_{k}}-2+T_{h, \ell_{-k}}\right) v_{i j}\right| \leq N\left(v_{\gamma_{0}}^{-}\right)^{2} .
$$

Next,

with

$$
I_{32}=N^{*} h^{2} v_{i j}^{-} \sqrt{a_{k}}\left|\Delta_{k} v_{i j}\right|=I_{321}+I_{322},
$$

$$
\begin{gathered}
I_{321}=N_{1}^{*} h^{2} \mu v_{i j}^{-} \sqrt{a_{k}} \Delta_{k} v_{i j}, \\
I_{322}=N^{*} h^{2} v_{i j}^{-} \sqrt{a_{k}}\left(\Delta_{k} v_{i j}\right)^{-} \leq N^{*} h^{2} v_{i j}^{-} \sqrt{a_{k}}\left|\Delta_{k} v_{i j}^{-}\right| \\
=N^{*} h v_{i j}^{-} \sqrt{a_{k}}\left|\delta_{k} v_{i j}^{-}+\delta_{-k} v_{i j}^{-}\right| \leq N^{*} \bar{W}_{1}+(1 / 3) \mu a_{k} R_{k}^{\mu},
\end{gathered}
$$

where the last inequality is true since $h v_{i j}^{-} \leq 2 \bar{W}_{1}^{1 / 2}$. Also observe that

$$
I_{321}=N_{1}^{*} h^{2} \sqrt{a_{k}} Z_{k}-N_{1}^{*} h^{2} v_{\gamma_{0}}^{-} \sqrt{a_{k}} \Delta_{k} v_{\gamma_{0}}+N_{1}^{*} h^{2} \nu v_{i} \sqrt{a_{k}} \Delta_{k} v_{i},
$$


where

$$
N_{1}^{*} \nu h^{2} v_{i} \sqrt{a_{k}} \Delta_{k} v_{i}=2 N_{1}^{*} \nu h v_{i} \sqrt{a_{k}} v_{k i} \leq N^{*} \nu^{2} h^{2} \bar{W}_{1}+a_{k} R_{k}^{\nu}
$$

and, according to (6.12) and the inequality $h\left|v_{\gamma_{0}}\right| \leq N \bar{W}_{1}^{1 / 2}$,

$$
-N_{1}^{*} h^{2} \sqrt{a_{k}} v_{\gamma_{0}}^{-} \Delta_{k} v_{\gamma_{0}} \leq N^{*} \bar{W}_{1}+(1 / 3) a_{k} R_{k}^{\gamma}
$$

Therefore,

$$
I_{321} \leq N_{1}^{*} h^{2} \sqrt{a_{k}} Z_{k}+N^{*}\left(1+\nu^{2} h^{2}\right) \bar{W}_{1}+(1 / 3) a_{k} R_{k}^{\gamma}+a_{k} R_{k}^{\nu} .
$$

We can now specify $h^{*}$ : we take

$$
N_{1}^{*} h^{*} \leq 1 \text { and } h^{*} \leq h_{0} / 2 .
$$

Then, for $h \leq h^{*}$,

$$
\begin{gathered}
I_{32} \leq h \sqrt{a_{k}} Z_{k}+N^{*} \nu^{2} \bar{W}_{1}+(1 / 3) \mu a_{k} R_{k}^{\mu}+(1 / 3) a_{k} R_{k}^{\gamma}+a_{k} R_{k}^{\nu}, \\
\left|4 I_{3}\right| \leq h \sqrt{a_{k}} Z_{k}+N^{*} \nu^{2} \bar{W}_{1}+N\left(v_{\gamma_{0}}^{-}\right)^{2} \\
+(1 / 3) a_{k} R_{k}^{\gamma}+(1 / 3) \mu a_{k} R_{k}^{\mu}+N^{*} a_{k} R_{k}^{\nu} .
\end{gathered}
$$

Estimating $I_{4}$. By using Lemma 6.2 we easily see that

$$
\left|4 I_{4}\right| \leq N\left(v_{\gamma_{0}}^{-}\right)^{2}+N^{*} \bar{W}_{1}+\nu \bar{W}_{1}^{1 / 2}\left(N \sqrt{a_{k}}+N^{*} h\right) \sum_{i}\left|v_{k i}\right|,
$$

where

$$
\begin{gathered}
N \nu \bar{W}_{1}^{1 / 2} \sqrt{a_{k}} \sum_{i}\left|v_{k i}\right| \leq N \nu \bar{W}_{1}+(1 / 3) \nu a_{k} R_{k}^{\nu} \\
N^{*} \nu \bar{W}_{1}^{1 / 2} h \sum_{k, i}\left|v_{k i}\right| \leq N^{*} \nu \bar{W}_{1} .
\end{gathered}
$$

It follows that

$$
\left|4 I_{4}\right| \leq N\left(v_{\gamma_{0}}^{-}\right)^{2}+N^{*} \nu \bar{W}_{1}+(1 / 3) \nu a_{k} R_{k}^{\nu} .
$$

By combining this with (6.11), (6.13), and (6.14), recalling that $Z_{k} \geq 0$, and coming back to (6.10) we conclude that

$$
4 J \geq\left(2 a_{k}-N_{1} h \sqrt{a_{k}}\right) Z_{k}-N\left(v_{\gamma_{0}}^{-}\right)^{2}-N^{*} \nu^{2} \bar{W}_{1}+\left(\nu / 3-N_{2}^{*}\right) a_{k} R_{k}^{\nu} .
$$

Now we specify $\nu=\nu^{*}$ by setting

$$
\nu^{*}=1+3 N_{2}^{*}
$$

and finish the argument as in [13. Namely, if $2 a_{k}-N_{1} h \sqrt{a_{k}} \geq 0$ for a $k$, then we can drop the term on the right in (6.15) corresponding to this $k$ because $Z_{k} \geq 0$. However, if $2 a_{k}-N_{1} h \sqrt{a_{k}} \leq 0$, then $\sqrt{a_{k}} \leq N h$ and $\left|a_{k}-N_{1} h \sqrt{a_{k}}\right| \leq N h^{2}$, whereas

$$
\begin{gathered}
h^{2} Z_{k}=h^{2}\left(v_{\gamma_{0}}^{-} \Delta_{k} v_{\gamma_{0}}+\mu v_{i j}^{-} \Delta_{k} v_{i j}-\nu v_{i} \Delta_{k} v_{k i}\right) \\
\leq N \max _{Q} W_{2}+N \nu \bar{W}_{1}^{1 / 2} \max _{Q} W_{2}^{1 / 2} \\
\leq N \max _{Q} W_{2}+N \nu^{2} \bar{W}_{1} \leq N\left(v_{\gamma_{0}}^{-}\right)^{2}+N^{*} \bar{W}_{1} .
\end{gathered}
$$

This and (6.15) yield (6.9) and the lemma is proved. 
Proof of Theorem 6.1. Fix a constant $\nu$ according to Lemma 6.5 and first assume that $\left(t_{0}, x_{0}\right) \in \partial_{2} Q$. Then

$$
\sqrt{\mu} \max _{\bar{Q}} W_{2}^{1 / 2} \leq V_{\gamma_{0} \mu \nu}^{1 / 2}\left(t_{0}, x_{0}\right) \leq N^{*}\left(\max _{\partial_{2} Q, i, j}\left|v_{i j}\right|+\max _{\partial_{2} Q, i}\left|v_{i}\right|\right),
$$

which by (6.4) yields a similar estimate for

$$
\max _{Q_{1}^{o}, i, j}\left|v_{i j}\right| .
$$

After that (6.2) is immediate (cf. the end of the proof of Theorem 4.1).

Therefore, in the rest of the proof we assume that

$$
\left(t_{0}, x_{0}\right) \in Q_{2}^{o} \text {. }
$$

Similarly, if (6.5) is violated, there is nothing to prove. Hence, we may assume that (6.5) holds. Finally, we may assume that $h \leq h^{*}$, where $h^{*}$ is taken from Lemma 6.5 and further reduced if needed so as to satisfy $h^{*} \sqrt{\nu} \leq \varepsilon$. Indeed, if $h \geq h^{*}$, then in $Q_{1}^{o}$,

$$
\left|v_{i j}\right| \leq 2\left(h^{*}\right)^{-1} \max _{Q}\left|v_{i}\right| .
$$

After justifying these additional assumptions, which allow us to use the assertions of Lemmas 6.2 and 6.5 as long as $h \leq h^{*}$, we construct functions $\bar{r}(t), \bar{a}_{k}(t, x)$, $\bar{b}_{k}(t, x), \bar{c}(t, x), \bar{f}(p, \psi, t, x)=\bar{f}(t, x)$ as in Section 4 to get (4.2) and (4.3) satisfied. Then, since $\left(t_{0}, x_{0}\right) \in Q_{2}^{o}$ and (6.3) and (6.5) are valid and $h \sqrt{\nu} \leq \varepsilon$, by Lemma 6.2 at $\left(t_{0}, x_{0}\right)$ we obtain

$$
P_{\gamma_{0} \mu \nu}\left(e^{-m \tau} \bar{r} \delta_{\tau}^{T} v+\bar{a}_{k} \Delta_{k} v+\bar{b}_{k} \delta_{k} v-\left(\bar{c}+\bar{r} c_{m}\right) v+\xi \bar{f}\right) \leq 0 .
$$

The fact that $\bar{r}, \bar{a}_{k}$, and $\bar{b}_{k}$ are limits of some $r^{\alpha}, a_{k}^{\alpha}$, and $b_{k}^{\alpha}$, allows us to assert that Lemma 6.5 holds with $\bar{r}, \bar{a}_{k}$, and $\bar{b}_{k}$ in place of $r^{\alpha}, a_{k}^{\alpha}$, and $b_{k}^{\alpha}$, respectively. Therefore,

$$
-N\left(v_{\gamma_{0}}^{-}\right)^{2}+P_{\gamma_{0} \mu \nu}\left(e^{-m \tau} \bar{r} \delta_{\tau}^{T} v-\left(\bar{c}+\bar{r} c_{m}\right) v+\xi \bar{f}\right) \leq N^{*} \bar{W}_{1} .
$$

Here $P_{\gamma_{0} \mu \nu} \delta_{\tau}^{T} v \geq 0$ as right after (4.6). Furthermore,

$$
P_{\gamma_{0} \mu \nu}\left(\left(\bar{c}+\bar{r} c_{m}\right) v\right)=-\left(\bar{c}+\bar{r} c_{m}\right) V_{\gamma_{0} \mu \nu}+I_{1},
$$

where $I_{1}$ is a linear combination of products of two types:

(i) $v_{\gamma_{0}}^{-}$or $v_{i j}^{-}$times a difference operator applied to $\bar{c}$ times either $v$ or a firstorder difference operator applied to $v$; the second and third factors may be taken at a point different from $\left(t_{0}, x_{0}\right)$, but their coefficients in the linear combination are dominated by a constant $N$;

(ii) $v_{i}$ times a difference operator applied to $\bar{c}$ times either $v$ or a first-order difference operator applied to $v$; these terms may be taken at a point different from $\left(t_{0}, x_{0}\right)$, but the coefficients of these terms are dominated by a constant $N^{*}$ (recall that $\nu$ is entering $\left.P_{\gamma \mu \nu}\right)$.

Owing to Lemma 6.2, the absolute value of the linear combination of the products of type (i) is less than

$$
N v_{\gamma_{0}}^{-} K_{3}\left(\max _{Q}|v|+\bar{W}_{1}^{1 / 2}\right) \leq\left(v_{\gamma_{0}}^{-}\right)^{2}+N^{*}\left(\max _{Q}|v|^{2}+\bar{W}_{1}\right) .
$$

The absolute value of the linear combination of the products of type (ii) is clearly less than

$$
N^{*} \bar{W}_{1}^{1 / 2}\left(\max _{Q}|v|+\bar{W}_{1}^{1 / 2}\right) \leq N^{*}\left(\max _{Q}|v|^{2}+\bar{W}_{1}\right) .
$$


Now from the above estimates, (6.16), and the fact that $V_{\gamma_{0} \mu \nu} \geq\left(v_{\gamma_{0}}^{-}\right)^{2}$ and $\bar{c}+\bar{r} c_{m} \geq \lambda$ we conclude that

$$
(\lambda-N)\left(v_{\gamma_{0}}^{-}\right)^{2}+P_{\gamma_{0} \mu \nu}(\xi \bar{f}) \leq N^{*}\left(\max _{Q}|v|^{2}+\bar{W}_{1}\right) .
$$

Finally, obviously

$$
P_{\gamma_{0} \mu \nu}(\xi \bar{f}) \geq-N^{*} \xi\left(\max _{i, j}\left|v_{i j}\right|+\nu \max _{i}\left|v_{i}\right|\right) \geq-N^{*} \xi^{2}-\left(v_{\gamma_{0}}^{-}\right)^{2}-\bar{W}_{1},
$$

and we infer from (6.17) that

$$
\left(\lambda-N_{1}\right)\left(v_{\gamma_{0}}^{-}\right)^{2} \leq N^{*}\left(\max _{Q}|v|^{2}+\bar{W}_{1}\right)+N^{*} \xi^{2} .
$$

We set the constant $N$ in the statement of the theorem to be $N_{1}+1$ and use Lemma 6.2 to conclude that in $\left.Q_{1}^{o} \cap Q\right|_{0}$ for any $i, j$,

$$
\left|\delta_{j} \delta_{i} u\right|=\left|v_{i j}\right| \leq N v_{\gamma_{0}}^{-}\left(t_{0}, x_{0}\right) \leq N^{*}\left(\max _{Q}|v|+\bar{W}_{1}^{1 / 2}\right)+N^{*} \xi\left(t_{0}\right) .
$$

This implies (6.2) in $\left.Q_{1}^{o} \cap Q\right|_{0}$. On the remaining part of $\left.Q\right|_{0}$ estimate (6.2) is obvious and the theorem is proved.

\section{Proof of Theorem 2.12}

We start with three auxiliary results. Everywhere in this section the assumptions of Theorem 2.12 are supposed to be satisfied. Recall that the set $\mathcal{L}$ is introduced in (2.20).

Lemma 7.1. For any function $\phi$ and $l_{1}, l_{2} \in \Lambda_{0}$ we have

$$
\begin{gathered}
\left|\delta_{h, l_{1}} \delta_{h, l_{2}} \phi(0)\right| \leq 4 \max \left(\left|\Delta_{h, \ell_{k}} \phi(x)\right|:|k| \leq d_{0}, x \in\left(\Lambda_{0}+\mathcal{L}\right) \cup\{0\}\right) \\
+4 \max \left(\left|\Delta_{h, \ell_{k}} \phi(x)\right|: d_{0}<|k| \leq d_{1}, x \in \Lambda_{0} \cup\{0\}\right) .
\end{gathered}
$$

Proof. Obviously we may assume that $h=1$. Next, observe that

$$
\begin{gathered}
\delta_{1, l_{1}} \delta_{1, l_{2}} \phi(0)=(1 / 2)\left[\Delta_{1, l_{2}} \phi\left(l_{1}\right)+\Delta_{1, l_{1}} \phi\left(l_{2}\right)\right]-(1 / 2) \Delta_{1, l_{1}-l_{2}} \phi(0), \\
\delta_{1, l_{1}} \delta_{1, l_{1}} \phi(0)=\Delta_{h, l_{1}} \phi\left(l_{1}\right), \quad \delta_{1, l_{1}} \delta_{1,-l_{1}} \phi(0)=-\Delta_{h, l_{1}} \phi(0) .
\end{gathered}
$$

It follows that if $l_{1}, l_{2} \in \mathcal{L}$, then

$$
\begin{gathered}
\left|\delta_{h, l_{1}} \delta_{h, l_{2}} \phi(0)\right| \leq \max \left(\left|\Delta_{h, \ell_{k}} \phi(x)\right|:|k| \leq d_{0}, x \in \mathcal{L} \cup\{0\}\right) \\
+\max \left(\left|\Delta_{h, \ell_{k}} \phi(0)\right|: d_{0}<|k| \leq d_{1}\right) .
\end{gathered}
$$

We substitute here $\phi(y+\cdot)$ in place of $\phi$ and use that $\Lambda_{0}+\mathcal{L} \supset \mathcal{L}$ since $d_{1} \geq 2$ and $\mathcal{L}=-\mathcal{L}$. Then we see that, if $y \in \Lambda_{0} \cup\{0\}$ and $l_{1}, l_{2} \in \mathcal{L}$, then

$$
\begin{gathered}
\left|\delta_{1, l_{1}} \delta_{1, l_{2}} \phi(y)\right| \leq \max \left(\left|\Delta_{h, \ell_{k}} \phi(x)\right|:|k| \leq d_{0}, x \in\left(\Lambda_{0}+\mathcal{L}\right) \cup\{0\}\right) \\
+\max \left(\left|\Delta_{h, \ell_{k}} \phi(x)\right|: d_{0}<|k| \leq d_{1}, x \in \Lambda_{0} \cup\{0\}\right) .
\end{gathered}
$$

In case $l_{1}=\zeta_{1}+\zeta_{2}, l_{2}=\eta_{1}+\eta_{2}$ with $\zeta_{1}, \zeta_{2}, \eta_{1}, \eta_{2} \in \mathcal{L}$ and $\zeta_{1} \neq \zeta_{2}, \zeta_{1} \neq-\zeta_{2}$, $\eta_{1} \neq \eta_{2}, \eta_{1} \neq-\eta_{2}$ either $\zeta_{1} \neq \eta_{1}$ and $\zeta_{1} \neq-\eta_{1}$ or $\zeta_{1} \neq \eta_{2}$ and $\zeta_{1} \neq-\eta_{2}$. The second possibility reduces to the first one by interchanging $\eta_{1}$ and $\eta_{2}$. If the first possibility is realized, then we use the formula $\delta_{1, \eta+\zeta}=T_{1, \eta} \delta_{1, \zeta}+\delta_{1, \eta}$ to obtain

$$
\begin{gathered}
\delta_{1, l_{1}} \delta_{1, l_{2}} \phi(0)=\left(T_{1, \zeta_{1}} \delta_{1, \zeta_{2}}+\delta_{1, \zeta_{1}}\right)\left(T_{1, \eta_{1}} \delta_{1, \eta_{2}}+\delta_{1, \eta_{1}}\right) \phi(0) \\
=\delta_{1, \zeta_{2}} \delta_{1, \eta_{2}} \phi\left(\zeta_{1}+\eta_{1}\right)+\delta_{1, \zeta_{2}} \delta_{1, \eta_{1}} \phi\left(\zeta_{1}\right)+\delta_{1, \zeta_{1}} \delta_{1, \eta_{2}} \phi\left(\eta_{1}\right)+\delta_{1, \zeta_{1}} \delta_{1, \eta_{1}} \phi(0) .
\end{gathered}
$$

Here $\zeta_{1}+\eta_{1} \in \Lambda_{0}, \zeta_{1}, \eta_{1} \in \Lambda_{0}$, and $0 \in \Lambda_{0} \cup\{0\}$. Therefore, we get (7.1) from (7.2). 
The remaining case that $l_{1} \in \mathcal{L}$ and $l_{2}=\eta_{1}+\eta_{2}$ with $\eta_{i}$ as above is taken care of by setting $\zeta_{1}=0$ in the above calculations. The lemma is proved.

Before stating the next lemma we remind the reader that the index $k$ takes values in $\left\{ \pm 1, \ldots, \pm d_{1}\right\}$.

Lemma 7.2. For any values of the arguments and $s>0$ we have

$$
\begin{aligned}
\sum_{k} q_{k}^{+} & \leq \frac{2 d_{1}}{\delta}\left[s^{-1} F\left(s \phi, s q_{k}, s p_{k}, s \psi\right)+K_{0} \sum_{k} q_{k}^{-}\right. \\
& \left.+K_{3}\left(\sum_{k}\left|p_{k}\right|+|\psi|+\phi^{-}+s^{-1}\right)\right] .
\end{aligned}
$$

Indeed, the expression in the brackets obviously is larger than

$$
\sup _{\alpha \in A} a_{k}^{\alpha}(t, x) q_{k}^{+},
$$

which in turn is larger than $\delta q_{n}^{+}$for each particular $n= \pm 1, \ldots, \pm d_{1}$.

Below we use the notation $\Gamma(\varepsilon)$ and $P_{\gamma}$ from Section 6 .

Lemma 7.3. Let $\theta \in\left(0, \varepsilon^{-1}\right)$ and $\varepsilon \in(0,1]$ be such that

$$
2 d_{1} K_{0} \kappa / \delta \leq 1 / 2, \quad \kappa:=\varepsilon(\theta+\varepsilon) /(1-\theta \varepsilon) .
$$

Let $w, \psi, p_{k}$ be functions on $Q$ and assume that

$$
\max _{\Gamma(\varepsilon) \times Q}\left(\xi P_{\gamma} w\right)^{-} \leq \theta \max _{Q} \sum_{k}\left|\xi \Delta_{h, \ell_{k}} w\right| .
$$

Then in $Q$ we have

$$
\sum_{k}\left|\xi \Delta_{h, \ell_{k}} w\right| \leq \frac{4 d_{1}}{\delta}(1+\kappa) I
$$

where

$$
I=\max _{Q}\left[\xi F\left(\phi, \Delta_{h, \ell_{k}} w, p_{k}, \psi\right)+K_{3} \xi\left(\sum_{|k| \leq d_{1}}\left|p_{k}\right|+|\psi|+\phi^{-}+1\right)\right] .
$$

Proof. Set $\Phi^{ \pm}=\sum_{k}\left(\xi \Delta_{h, \ell_{k}} w\right)^{ \pm}$and observe that due to (7.4),

$$
\varepsilon \Phi^{+}-\varepsilon^{-1} \Phi^{-} \geq-\theta \max _{Q} \Phi^{+}-\theta \max _{Q} \Phi^{-}
$$

in $Q$. Hence $(\theta+\varepsilon) \max _{Q} \Phi^{+} \geq\left(\varepsilon^{-1}-\theta\right) \max _{Q} \Phi^{-}$, that is,

$$
\max _{Q} \Phi^{-} \leq \kappa \max _{Q} \Phi^{+} \text {. }
$$

By (7.5) and Lemma 7.2 with $s^{-1} \phi, s^{-1} \Delta_{h, \ell_{k}} w, s^{-1} p_{k}$, and $s^{-1} \psi$ in place of $\phi, q_{k}, p_{k}$, and $\psi$, respectively, and $s^{-1}=\xi$ we find that in $Q$,

$$
\Phi^{+} \leq \frac{2 d_{1}}{\delta}\left[I+K_{0} \kappa \max _{Q} \Phi^{+}\right]
$$

Upon taking the maximums over $Q$ of both parts and taking into account (17.3) we get that $\Phi^{+} \leq\left(4 d_{1} / \delta\right) I$ in $Q$, which along with (7.5) yields the result. The lemma is proved. 
Proof of Theorem 2.12. Here $k, i, j$ run through $\pm 1, \ldots, \pm d_{1}$. It is easy to see that one can find $\varepsilon=\varepsilon\left(\delta, d_{1}, K_{0}\right) \in(0,1]$ and $\mu=\mu\left(\delta, d_{1}, K_{0}\right)>0$ in such a way that the conditions:

$$
16 d_{1}^{2} \mu \leq \varepsilon^{2}, \quad 16 d_{1}^{2}(3 \mu)^{1 / 2}=: \theta<\varepsilon^{-1}
$$

and (7.3) are satisfied. We choose and fix appropriate $\varepsilon$ and $\mu$.

If

$$
\max _{\Gamma(\varepsilon) \times Q}\left(\xi P_{\gamma} u\right)^{-} \leq \theta \max _{Q} \sum_{k}\left|\xi \Delta_{h, \ell_{k}} u\right|,
$$

then, by taking into account that $u$ satisfies (2.1) in $Q$, from Lemma 7.3 we obtain that $\left|\xi \Delta_{h, \ell_{k}} u\right|$ are bounded in $Q$ by the right-hand side of (2.21). By combining this with Lemma 7.1 we conclude that (2.21) is true in $\left.Q\right|_{0} \cap Q_{2}^{o}$ (notice that $\left.\left(\Lambda_{0}+\mathcal{L}\right) \cup\{0\} \subset \Lambda_{0}+\Lambda_{0}\right)$. Of course, (2.21) is obvious on $\left.Q\right|_{0} \cap \partial_{2} Q$.

If (6.1) holds, then we get (2.21) from Theorem 6.1. In the remaining case both (7.6) and (6.1) are violated and

$$
\begin{gathered}
\theta H:=\theta \max _{Q} \sum_{k}\left|\xi \Delta_{h, \ell_{k}} u\right| \\
\leq \max _{\Gamma(\varepsilon) \times Q}\left(\xi P_{\gamma} u\right)^{-} \leq(3 \mu)^{1 / 2} \max _{Q}\left(\sum_{i, j}\left|\xi \delta_{h, \ell_{j}} \delta_{h, \ell_{i}} u\right|^{2}\right)^{1 / 2} \\
\leq(3 \mu)^{1 / 2} \max _{\partial_{2} Q} \sum_{i, j}\left|\xi \delta_{h, \ell_{j}} \delta_{h, \ell_{i}} u\right|+(3 \mu)^{1 / 2} \max _{Q_{2}^{o}} \sum_{i, j}\left|\xi \delta_{h, \ell_{j}} \delta_{h, \ell_{i}} u\right| .
\end{gathered}
$$

In light of Lemma 7.1 the last maximum over $Q_{2}^{o}$ is less than $8 d_{1}^{2} H$. Hence

$$
\theta H \leq N \max _{\partial_{2} Q, i, j}\left|\xi \delta_{h, \ell_{j}} \delta_{h, \ell_{i}} u\right|+(1 / 2) \theta H, \quad H \leq N \max _{\partial_{2} Q, i, j}\left|\xi \delta_{h, \ell_{j}} \delta_{h, \ell_{i}} u\right|,
$$

and we can finish the proof of (2.21) in the same way as we did a few times before. The theorem is proved.

\section{Proof of Theorem 2.14}

In the following lemma the assumption that $\sup _{\alpha} a_{k}^{\alpha} \geq \delta$ is not used. All other assumptions of Theorem 2.14 are supposed to hold. We use notation (2.10) and the notation from Section 6 with $d_{1}+1$ in place of $d_{1}$ and $\delta_{i}=\delta_{h_{i}, \ell_{i}}, \Delta_{i}=\Delta_{h_{i}, \ell_{i}}$. Here we take

$$
\mu=0
$$

and show how to choose $\nu=\nu\left(\lambda, \varepsilon, d_{1}, K_{3}\right)$ in Lemma 8.1.

Lemma 8.1. In $\left.Q\right|_{0}$ for $\gamma \in \Gamma(\varepsilon)$ we have

$$
\begin{gathered}
\left(\sum_{i} \gamma_{i} \Delta_{h_{i}, \ell_{i}} u\right)^{-} \leq N^{*} e^{m^{+}(T+\tau)}\left(1+\max _{\bar{Q}, j}\left|\xi_{(-)} \delta_{h_{j}, \ell_{j}} u\right|\right. \\
\left.+\max _{\bar{Q}}\left|\xi_{(-)} u\right|+\max _{\partial_{1} Q, j}\left(\xi_{(-)} \Delta_{h_{j}, \ell_{j}} u\right)^{-}\right),
\end{gathered}
$$

where $N^{*}=N^{*}\left(\lambda, h_{0}, \varepsilon, d_{1}, K_{3}\right)$.

Proof. As many times before, if $\left(t_{0}, x_{0}\right) \in \partial_{1} Q$, there is nothing to prove. Therefore, we assume that $\left(t_{0}, x_{0}\right) \in Q_{1}^{0}$. We may also assume that at $\left(t_{0}, x_{0}\right)$,

$$
\sum_{i}\left(\xi \delta_{h_{i}, \ell_{i}} u\right)^{2} \leq\left[\left(\xi \sum_{i} \gamma_{0 i} \Delta_{h_{i}, \ell_{i}} u\right)^{-}\right]^{2}
$$


Then the operator $P_{\gamma_{0} 0 \nu}$ respects the maximum principle for $h \nu \leq 2 \varepsilon$ (see the proof of Lemma 6.2 and recall that $\eta \leq h$ ).

Then as in the proof of Lemma 6.5 we obtain

$$
4 J=4 P_{\gamma_{0} 0 \nu}\left(a_{k} \Delta_{k} v+b_{k} \delta_{k} v\right) \geq a_{k} \nu R_{k}^{\nu}+4 I_{4}
$$

if $h \leq h_{0} / 2$, where this time

$$
I_{4}=2 v_{\gamma_{0}}^{-} \gamma_{0 i}\left(\delta_{i} b_{k}\right) v_{k i}+v_{\gamma_{0}}^{-} \gamma_{0 i}\left(\Delta_{i} b_{k}\right) v_{k}-\nu v_{i}\left(\delta_{i} b_{k}\right) T_{h_{i}, \ell_{i}} v_{k} .
$$

Since

$$
v_{\gamma_{0}}^{-} \gamma_{0 i}\left|\left(\delta_{i} b_{k}\right) v_{k i}\right| \leq N^{*} v_{\gamma_{0}}^{-} \sqrt{a_{k}} \sum_{i}\left|v_{k i}\right| \leq(\lambda / 8)\left(v_{\gamma_{0}}^{-}\right)^{2}+N^{*} a_{k} \sum_{i} v_{k i}^{2}
$$

we get from (8.2) that, for $\nu=\nu^{*}\left(\lambda, \varepsilon, d_{1}, K_{3}\right), h \nu^{*} \leq 2 \varepsilon$, and $h \leq h_{0} / 2$,

$$
J \geq-(\lambda / 2)\left(v_{\gamma_{0}}^{-}\right)^{2}-N^{*} \bar{W}_{1} .
$$

The rest is just a repetition of a part of the proof of Theorem 6.1 with obvious and great simplifications. The lemma is proved.

There is almost nothing else to do to finish the proof of Theorem 2.14 Indeed, (8.1) with $d_{1}$ in place of $d_{1}+1$ yields the first estimate in (2.22) as in the proof of Theorem 2.12. After getting estimates for $\left|\Delta_{h, \ell_{k}} u\right|$ the estimate of $\left(\Delta_{\eta, l} u\right)^{-}$follows immediately from (8.1). The theorem is proved.

\section{Comments on the operators having Form (2.7)}

We know (see, for instance, [5]) that if an operator $L$ having form (2.7) admits an approximation with operators $S_{h}$ of the form (2.4) respecting the maximum principle with $h B$ in place of $B$ and $\operatorname{Span} B=\mathbb{R}^{d}$, then necessarily

$$
L u=a_{k} \ell_{k}^{i} \ell_{k}^{j} u_{x^{i} x^{j}}+b_{k} \ell_{k}^{i} u_{x^{i}}
$$

with some $a_{k}, b_{k} \geq 0$ and $\ell_{k} \in B \cup(-B)$. A way to find such representations for $d=2$ and given $a^{i j}$ is suggested in [1].

The next natural issue is related to the smoothness of $a_{k}, b_{k}$ if we are given that the $a^{i j}$ are smooth. Recall that in Assumption 2.2 we need $a_{k}^{\alpha}$ to be at least Lipschitz continuous. Of course, this problem disappears if the $a^{i j}$ are constant.

It is an easy and probably well-known fact that if $\left(a^{i j}\right)=\left(a^{i j}(t, x)\right)$ is uniformly bounded and uniformly elliptic, then one can find $d_{1}$ and $\Lambda_{0}$ for which $a_{k}$ can be chosen strictly positive and as smooth as $a^{i j}$ are. The proof of this can be obtained from the fact that if we are given a closed convex polyhedron, then every point in the relative interior can be written as a convex combination of the extreme points with the coefficients $>0$ which are infinitely differentiable functions of the point. By replacing $L$ with $L+\varepsilon^{2} \Delta$ one can approximate a possibly degenerate operator $L$ with uniformly nondegenerate ones, so that there always exist a sequence of operators of the form (2.7) approximating $L$. Notice, however, that generally the set $\Lambda_{0}$ changes with $\varepsilon$. Nevertheless, one knows how to estimate the difference of solutions corresponding to $L+\varepsilon^{2} \Delta$ and $L$ (see, for instance, [9]) and between the solutions of the corresponding finite-difference approximations (see, for instance, Theorem 5.6 of [13] or Remark 2.6).

Another generic example is given by the so-called diagonally dominant matrices. For instance, take $d=2$ and assume that $b \equiv 0$, the $a^{i j}$ are twice continuously differentiable with respect to $x, a^{12}=a^{21}$, and $\left|a^{12}\right| \leq s$, where $s=a^{11} \wedge a^{22}$. 
Set $\kappa=1 / 3$ and take an infinitely differentiable, even, and convex function $\psi(t)$ on $\mathbb{R}$ such that $\psi(y)=|y|$ for $|y| \geq \kappa$. Introduce

$$
g=a^{12} s^{-1}, \quad h=s \psi(g), \quad 2 \hat{a}^{1, \pm 2}=h \pm a^{12}, \quad 2 \hat{a}^{i i}=a^{i i}-h,
$$

where $i=1,2$ and $0 \cdot 0^{-1}:=0$. For other values of $i, j= \pm 1, \pm 2$ define $\hat{a}^{i j}$ so that

$$
\hat{a}^{i j}=\hat{a}^{j i}, \quad \hat{a}^{-i,-j}=\hat{a}^{i j}, \quad \hat{a}^{i,-i}=0
$$

and set

$$
\ell_{j}=e_{|j|} \operatorname{sign} j, \quad \ell_{i j}=\ell_{i}+\ell_{j},
$$

where $e_{1}, e_{2}$ are the basis vectors. Then simple manipulations yield

$$
4 L u=\hat{a}^{i j} \ell_{i j}^{k} \ell_{i j}^{r} u_{x^{k} x^{r}}
$$

We now show that not only does $L$ admit a representation as the sum of secondorder directional derivatives with the directions independent of $t, x$ but also the $\sqrt{\hat{a}^{i j}}$ are Lipschitz continuous in $x$. By the way, observe that obviously $\hat{a}^{i j} \geq 0$.

We are going to use that nonnegative and twice continuously differentiable functions are the squares of Lipschitz continuous functions. In particular, the $a^{i i}$ and, consequently, $a^{11} \wedge a^{22}$ are the squares of Lipschitz continuous functions, $\left|a_{x}^{i i}\right| \leq N \sqrt{a^{i i}}$ and $\left|s_{x}\right| \leq N \sqrt{s}$. Furthermore, $a^{11} \pm a^{12}$ is nonnegative and twice continuously differentiable. Hence, it is the square of a Lipschitz continuous function. In particular,

$$
\left|a_{x}^{11} \pm a_{x}^{12}\right| \leq N \sqrt{a^{11} \pm a^{12}}, \quad\left|a_{x}^{12}\right| \leq N \sqrt{a^{11}}, \quad\left|a_{x}^{12}\right| \leq N \sqrt{s},
$$

and recalling that $\left|a^{12}\right| \leq s$ we find

$$
\left|g_{x}\right| \leq\left|a_{x}^{12}\right| s^{-1}+\left|a^{12}\right| \cdot\left|s_{x}\right| s^{-2} \leq N s^{-1 / 2}, \quad\left|h_{x}\right| \leq N \sqrt{s} .
$$

Next, the function $\phi(y):=\psi(y)+y$ is smooth and nonnegative. Therefore

$$
2\left|\hat{a}_{x}^{12}\right|=\left|\phi^{\prime} g_{x} s+\phi s_{x}\right| \leq N \sqrt{\phi} \sqrt{s}+N \phi \sqrt{s} \leq N \sqrt{\phi} \sqrt{s}=N \sqrt{\hat{a}^{12}} .
$$

A similar estimate holds for $\left|\hat{a}_{x}^{1,-2}\right|$ and $\left|\hat{a}_{x}^{i j}\right|$ if $i \neq j$.

On the set where $\left|a^{12}\right|>\kappa s$, we have $2 \hat{a}^{11}=a^{11}-\left|a^{12}\right|$, so that by the above,

$$
\left|\hat{a}_{x}^{11}\right|=\left|a_{x}^{11}-a_{x}^{12} \operatorname{sign} a^{12}\right| \leq N \sqrt{a^{11}-a^{12} \operatorname{sign} a^{12}}=N \sqrt{\hat{a}^{11}} .
$$

Finally, on the set where $\left|a^{12}\right|<2 \kappa s$, it holds that $h \leq 2 \kappa s, a^{11}-h \geq \kappa a^{11}$, and $\left|\hat{a}_{x}^{11}\right| \leq N \sqrt{a^{11}} \leq N \sqrt{\hat{a}^{11}}$. Similarly we get what we need for $\hat{a}^{22}$ and the remaining $\hat{a}^{i i}$.

\section{REFERENCES}

[1] Bonnans, J. F., Ottenwaelter, E., and Zidani, H., A fast algorithm for the two dimensional HJB equation of stochastic control, M2AN Math. Model. Numer. Anal., Vol. 38 (2004), No. 4, 723-735. MR2087732 (2005e:93165)

[2] Barles, G., and Jakobsen, E.R., On the convergence rate of approximation schemes for Hamilton-Jacobi-Bellman equations, Mathematical Modelling and Numerical Analysis, ESAIM, M2AM, Vol. 36 (2002), No. 1, 33-54. MR.1916291 (2003h:65142)

[3] Barles, G., and Jakobsen, E.R., Error bounds for monotone approximation schemes for Hamilton-Jacobi-Bellman equations, SIAM J. Numer. Anal., Vol. 43 (2005), No. 2, 540-558. MR2177879

[4] Barles, G., and Jakobsen, E.R., Error bounds for monotone approximation schemes for parabolic Hamilton-Jacobi-Bellman equations, preprint.

[5] Dong, Hongjie and Krylov, N.V., On the rate of convergence of finite-difference approximations for Bellman equations with constant coefficients, Algebra i Analiz (St. Petersburg Math. J.), Vol. 17 (2005), No. 2, 108-132. MR2159586 (2006f:49050) 
[6] Dong, Hongjie and Krylov, N.V., On the rate of convergence of finite-difference approximations for degenerate linear parabolic equations with $C^{1}$ and $C^{2}$ coefficients, Electron. J. Diff. Eqns., Vol. 2005 (2005), No. 102, pp. 1-25. http://ejde.math.txstate.edu MR2162263 (2006i:35008)

[7] Dong, Hongjie and Krylov, N.V., On the rate of convergence of finite-difference approximations for parabolic Bellman equations with Lipschitz coefficients in cylindrical domains, submitted to Applied Math. and Optimization.

[8] Jakobsen, E. R., On the rate of convergence of approximation schemes for Bellman equations associated with optimal stopping time problems, Math. Models Methods Appl. Sci., Vol. 13 (2003), No. 5, 613-644. MR.1978929 (2004g:49054)

[9] Krylov, N.V., "Controlled diffusion processes", Nauka, Moscow, 1977 in Russian; English translation Springer, 1980. MR601776 (82a:60062)

[10] Krylov, N.V., On the rate of convergence of finite-difference approximations for Bellman's equations, Algebra i Analiz, St. Petersburg Math. J., Vol. 9 (1997), No. 3, 245-256. MR:1466804 (98h:49033)

[11] Krylov, N.V., Approximating value functions for controlled degenerate diffusion processes by using piece-wise constant policies, Electronic Journal of Probability, Vol. 4 (1999), paper no. 2, 1-19, http://www.math.washington.edu/ ejpecp/EjpVol4/ paper2.abs.html. MR.1668597 (2000b:49056)

[12] Krylov, N.V., On the rate of convergence of finite-difference approximations for Bellman's equations with variable coefficients, Probab. Theory Relat. Fields, Vol. 117 (2000), No. 1, 1-16. MR1759507 (2001j:65134)

[13] Krylov, N.V., The rate of convergence of finite-difference approximations for Bellman equations with Lipschitz coefficients, Applied Math. and Optimization, Vol. 52 (2005), No. 3, 365-399. MR2174020 (2006k:65219)

Department of Mathematics, University of Minnesota, 127 Vincent Hall, MinneapoLiS, Minnesota 55455

E-mail address: krylov@math.umn.edu 